\title{
On Training Targets for Deep Learning Approaches to Clean Speech Magnitude Spectrum Estimation
}

\author{
Aaron Nicolson ${ }^{1, \mathrm{a}}$ and Kuldip K. Paliwal ${ }^{1, \mathrm{~b}}$ \\ Signal Processing Laboratory, Griffith University, Brisbane, Queensland 4111, Australia
}

The estimation of the clean speech short-time magnitude spectrum (MS) is key for speech enhancement and separation. Moreover, an automatic speech recognition (ASR) system that employs a front-end relies on clean speech MS estimation to remain robust. Training targets for deep learning approaches to clean speech MS estimation fall into three main categories: computational auditory scene analysis (CASA), MS, and minimum mean-square error (MMSE) training targets. In this study, we aim to determine which training target produces enhanced/separated speech at the highest quality and intelligibility, and which is most suitable as a front-end for robust ASR. The training targets were evaluated using a temporal convolutional network (TCN) on the DEMAND Voice Bank and Deep Xi datasetswhich include real-world non-stationary and coloured noise sources at multiple SNR levels. Seven objective measures were used, including the word error rate (WER) of the Deep Speech ASR system. We find that MMSE training targets produce the highest objective quality scores. We also find that CASA training targets, in particular the ideal ratio mask (IRM), produce the highest intelligibility scores and perform best as a front-end for robust ASR.

(C)2020 Acoustical Society of America. [https://doi.org(DOI number)]

$[\mathrm{XYZ}]$

Pages: $1-15$

\section{INTRODUCTION}

Speech corrupted by background noise (or noisy speech) can reduce the efficiency of communication between a speaker and a listener. The linguistic content of noisy speech can be misinterpreted by the listener or the background noise can cause fatigue. Factory welding, music, and non-target speakers are all examples of background noise sources (Loizou, 2013). The performance of speech processing systems, such as automatic speech recognition (ASR), automatic speaker verification (ASV), and automatic speaker identification (ASI) systems, can also be impacted by noisy speech (Le Prell and Clavier, 2017; Nicolson and Paliwal, 2020). A system that can remove background noise or segregate the target speech (or clean speech) is thus indispensable for a speech processing system. Speech corrupted by background noise and reverberation from surface reflections (or noisy-reverberant speech) and systems with multiple microphones pose a more difficult task for such systems (Tawara et al., 2019; Zhao et al., 2017). For simplicity, this study focuses on non-reverberant noisy speech recorded using a single-microphone (or single-channel noisy speech).

The objective of speech enhancement is to improve the perceived quality and intelligibility of noisy speech. Speech enhancement algorithms accomplish this task by suppressing background noise without distorting the speech (Loizou, 2013). They are used to suppress background noise during phone calls, conference calls, and are

a aaron.nicolson@griffithuni.edu.au

bk.paliwal@griffith.edu.au used in hearing aid devices. A popular approach is to use an estimator of the clean speech that is derived from statistical models and an optimisation criteria. The Wiener filter (WF) optimally estimates, in the mean-squared error (MSE) sense, the discrete Fourier transform (DFT) coefficients of the clean speech-when the clean speech and noise DFT coefficients are assumed to be independent Gaussian random variables (Lim and Oppenheim, 1979). The minimum mean-square error (MMSE) shorttime spectral amplitude (MMSE-STSA) and MMSE logspectral amplitude (MMSE-LSA) estimators differ from the WF by optimally estimating the clean speech magnitude and log-magnitude spectra, respectively (Ephraim and Malah, 1984, 1985). While the WF is a linear estimator that depends solely on the a priori signal-to-noise ratio (SNR), the MMSE-STSA and MMSE-LSA estimators are non-linear estimators that depend on both the a priori and a posteriori SNR. Other prominent estimators assume super-Gaussian clean speech and noise priors (Martin, 2005) or employ a perceptually motivated optimisation criteria (Loizou, 2005). In this study, we focus on the MMSE-STSA and MMSE-LSA estimators, and the WF, which are referred to collectively as MMSE estimators henceforth.

An alternative to speech enhancement is speech separation (or segregation) - a special case of source separation where the target speech is segregated from the noisy speech. Source separation is performed using computational auditory scene analysis (CASA) - the computational task of segregating mixtures of sound sources (Wang and Brown, 2006). One approach to speech separation is to classify each time-frequency (TF) component of the noisy speech as either speech or noise dominant 
(Wang, 2005). This is realised by using the ideal binary mask (IBM) as the objective of CASA, which reduces speech separation to a binary classification problem. The IBM can be used to attenuate the noise-dominant TF components. In practice, however, a noisy speech $\mathrm{TF}$ component can contain a ratio of speech and noise. Instead of a hard label, a soft label can be used to segregate the target speech in a noisy speech TF component (Srinivasan et al., 2006). This is realised by using the ideal ratio mask (IRM) as the objective of CASA, which has the same form as the square-root WF (SRWF) (Lim and Oppenheim, 1979).

While subjective listening tests often accurately evaluate the quality and intelligibility of enhanced/separated speech under stringent conditions, they are costly and time consuming (Hu and Loizou, 2008). Thus, objective measures of quality and intelligibility are often used as an alternative. For objective quality, the perceptual evaluation of speech quality (PESQ) measure is commonly used in the literature (Rix et al., 2001). However, PESQ was developed for the purpose of evaluating the distortions introduced by speech codecs and communication channels - not for the distortions introduced by speech enhancement/separation systems. In (Hu and Loizou, 2008), several composite objective quality measures were developed specifically for the evaluation of the signal distortion (CSIG), background noise intrusiveness (CBAK), and overall signal quality (COVL) of enhanced/separated speech. Their correlation with subjective scores was higher than that of previous objective quality measures, including PESQ. For objective intelligibility, the short-time objective intelligibility (STOI) measure is commonly used and demonstrates a high correlation with subjective scores (Taal et al., 2011). The word error rate (WER) of an ASR system can also be used to objectively evaluate the intelligibility of enhanced/separated speech (Thomas-Stonell et al., 1998).

Currently, deep learning approaches are at the forefront of speech enhancement and separation. Deep neural networks (DNNs) provide a non-linear map from a given noisy speech representation to a target representation. DNNs were first employed for IBM estimation, where a feedforward neural network (FNN) provided learned features to linear support vector machines (SVMs) (Wang and Wang, 2013). It was found that the use of a DNN enabled the system to generalise to unobserved speakers and noise sources. FNNs were later employed for IRM estimation, where it was found that an FNN-IRM estimator is able to outperfrom an FNN-IBM estimator when used as a front-end for ASR (Narayanan and Wang, 2013). An FNN was later used to estimate the clean speech magnitude spectrum (MS), which was found to outperform the combination of the MMSE-LSA estimator and the improved minima controlled recursive averaging (IMCRA) noise estimation approach (Cohen, 2003; Xu et al., 2015). In a study by Wang et al. (Wang et al., 2014), the IBM, IRM, MS, and spectral magnitude mask (SMM) were compared as training targets, where the SMM is the ratio of the clean speech MS to the noisy speech MS. It was found that the IRM and SMM as the training target produced higher objective quality and intelligibility scores than the IBM and the MS. However, the study was limited by its use of only PESQ and STOI as objective measures. In (Nicolson and Paliwal, 2019a), a deep learning approach to a priori SNR estimation improved the performance of MMSE estimators. It was found that the a priori SNR as the training target produces comparable results to the IRM when a residual long shortterm memory (ResLSTM) network was used (Kim et al., 2017). However, this study was also limited as objective scores were computed using only a wideband extension of PESQ (Morioka et al., 2005) and STOI.

The aforementioned training targets utilise only the magnitude of each TF component. However, there are a set of training targets that incorporate the phase of each TF component. The phase sensitive mask (PSM) is an extension of the SMM that includes the phase difference between each clean and noisy speech TF component (Erdogan et al., 2015). Results in (Williamson et al., 2016) indicate that the PSM is able to outperform the IRM. The complex IRM (cIRM) is a complex TF mask that utilises both the real and imaginary components of the target speech and noise DFT coefficients (Williamson et al., 2016). Results indicate that the cIRM is able to outperform the PSM and IRM in terms of objective quality and intelligibility. In (Pascual et al., 2017), clean speech time-domain samples were used as the training target. However, results in (Williamson et al., 2016) indicate that the PSM, cIRM, and IRM are able to attain higher objective quality and intelligibility scores than clean speech time-domain samples.

Current methods used to increase the robustness of an ASR system include 1) utilising a deep learning approach to speech enhancement/separation as a front-end for noisy speech pre-processing, and 2) multi-condition training (Zhang et al., 2018). It has been found that utilising multi-condition training or a deep learning-based speech enhancement/separation front-end significantly improves the robustness of an ASR system, with the combination of both methods providing the best performance (Narayanan and Wang, 2013). In this study, we concentrate solely on deep learning-based speech enhancement/separation front-ends. Features used as input to current ASR acoustic models are derived from the MS (Kriman et al., 2020; Moritz et al., 2020; Wang et al., 2020). These include mel-scale filterbank, gammatone filterbank, and cepstral-domain features (Schluter et al., 2007). When using a deep learning-based speech enhancement/separation front-end, such features are computed from the enhanced/separated speech MS (Nicolson and Paliwal, 2019b). This means that phase inclusive training targets procure no advantage when employed as a front-end for robust ASR.

The aim of this study is to determine which training target is best for clean speech MS estimation -in the context of speech enhancement/separation and robust ASR performance. Thus, we extend this research to include training targets that make use of the short-time phase 
spectrum in a future study. We also propose to jointly estimate the a priori and a posteriori SNR - to increase the performance of the MMSE-STSA and MMSE-LSA estimators. Temporal convolutional networks (TCNs) have been the DNN of choice for deep learning approaches to speech enhancement/separation as of late (Nikzad et al., 2020). Hence, each training target is evaluated using a residual network (ResNet) TCN that has recently been employed for speech enhancement. The DEMAND Voice Bank and Deep Xi datasets are included in the experiment setup, which include real-world non-stationary and coloured noise sources at multiple SNR levels. We also investigate which function for compressing the dynamic range of the training target values is best. We also assess which loss function is most appropriate for each training target. Multiple objective quality and intelligibility measures are included in the experiment setup, including CSIG, CBAK, COVL, PESQ, SegSNR, STOI, and WER.

In this paper, we first describe the analysis, modification, and synthesis (AMS) framework (Section II). MMSE estimators are then described in Section III. In Section IV, the training targets are described. The experiment setup is described in Section V. The results are discussed in Section VI. Conclusions are drawn in Section VII.

\section{ANALYSIS, MODIFICATION, \& SYNTHESIS FRAME- WORK}

The short-time Fourier analysis, modification, and synthesis (AMS) framework is used for speech enhancement/separation (Allen, 1977; Allen and Rabiner, 1977). The AMS framework consists of three stages: (1) the analysis stage, where noisy speech undergoes short-time Fourier transform (STFT) analysis; (2) the modification stage, where the noisy speech spectrum is modified; and (3) the synthesis stage, where the enhanced/separated speech is synthesised by applying the inverse STFT (ISTFT).

In the time-domain, the noisy speech signal, $x[n]$, is given by:

$$
x[n]=s[n]+d[n],
$$

where $s[n]$ and $d[n]$ denote the clean/target speech and uncorrelated additive noise, respectively, and $n$ denotes the discrete-time sample. The noisy speech is analysed frame-wise using the running STFT (Vary and Martin, 2006):

$$
X[l, k]=\sum_{n=0}^{N_{d}-1} x\left[n+l N_{s}\right] w[n] e^{-j 2 \pi n k / N_{d}},
$$

where $l$ denotes the time-frame index, $k$ denotes the discrete-frequency bin, $N_{d}$ denotes the time-frame duration in discrete-time samples, $N_{s}$ denotes the time-frame shift in discrete-time samples, and $w[n]$ is an analysis window function.

As we aim to modify the noisy speech MS, the polar form of the noisy speech spectrum is used:

$$
X[l, k]=|X[l, k]| e^{j \angle X[l, k]},
$$

where $|X[l, k]|$ and $\angle X[l, k]$ denote the noisy speech magnitude and phase spectra, respectively. Similarly, the clean speech magnitude and phase spectra are denoted as $|S[l, k]|$ and $\angle S[l, k]$, respectively, and the noise magnitude and phase spectra are denoted as $|D[l, k]|$ and $\angle D[l, k]$, respectively. The modified spectrum is constructed by combining an estimate of the clean speech MS, $|\hat{S}[l, k]|$, with the noisy speech phase spectrum:

$$
Y[l, k]=|\hat{S}[l, k]| e^{j \angle X[l, k]} .
$$

The synthesis stage involves applying the ISTFT to the modified spectrum. First, the inverse discrete Fourier transform is applied to the modified spectrum:

$$
y_{f}[l, n]=\frac{1}{N_{d}} \sum_{k=0}^{N_{d}-1} Y[l, k] e^{j 2 \pi n k / N_{d}}
$$

where $y_{f}[l, n]$ is the framed enhanced/separated speech. The overlap-add method is subsequently applied to produce the final enhanced/separated speech (Crochiere, 1980):

$$
y[n]=\frac{\sum_{l=-\infty}^{\infty} y_{f}\left[l, n-l N_{s}\right]}{\sum_{l=-\infty}^{\infty} w\left[n-l N_{s}\right]},
$$

where $w[n]$ is a synthesis window function. In this work, the Hamming window function is used for analysis and synthesis, with a frame-duration of $32 \mathrm{~ms}\left(N_{d}=512\right)$ and a frame-shift of $16 \mathrm{~ms}\left(N_{s}=256\right)$.

\section{MMSE ESTIMATORS}

The MMSE-LSA estimator of the clean speech MS is defined as

$$
|\hat{S}[l, k]|=|X[l, k]| \frac{\xi[l, k]}{\xi[l, k]+1} \exp \left\{\frac{1}{2} \int_{\nu[l, k]}^{\infty} \frac{e^{-t}}{t} d t\right\},
$$

where $\nu[l, k]=\frac{\xi[l, k]}{\xi[l, k]+1} \gamma[l, k], \xi[l, k]$ is the a priori SNR and $\gamma[l, k]$ is the a posteriori SNR. The a priori SNR is defined as

$$
\xi[l, k]=\frac{\lambda_{s}[l, k]}{\lambda_{d}[l, k]},
$$

where $\lambda_{s}[l, k]=\mathrm{E}\left\{|S[l, k]|^{2}\right\}$ is the variance of the clean speech spectral component, and $\lambda_{d}[l, k]=\mathrm{E}\left\{|D[l, k]|^{2}\right\}$ is the variance of the noise spectral component. The $a$ posteriori $\mathrm{SNR}$ is defined as

$$
\gamma[l, k]=\frac{|X[l, k]|^{2}}{\lambda_{d}[l, k]} .
$$

The SRWF is defined as

$$
|\hat{S}[l, k]|=|X[l, k]| \sqrt{\frac{\xi[l, k]}{\xi[l, k]+1}} .
$$




\section{TRAINING TARGETS}

For speech enhancement/separation, a DNN learns to map the noisy speech MS to a training target that can be used to estimate the clean speech MS in Equation (4). Training targets with magnitude or power values tend to be difficult for a DNN to learn, as noted in (Wang et al., 2014). Hence, a function must be used to compress their dynamic range - which we refer to as a compression function henceforth (previously referred to as a mapping function (Nicolson and Paliwal, 2019a)). Here, the training target is denoted as $t[l, k]$. The training target can be computed from the clean speech and noise of the noisy speech in Equation (1), as they are observed during training. The training targets described in this section belong to one of three categories: CASA, MS, and MMSE estimator training targets.

\section{A. CASA training targets}

The CASA training targets, including the IBM and IRM, are detailed here. The IBM is computed by applying a threshold to the instantaneous a priori SNR:

$$
t[l, k]=\operatorname{IBM}[l, k]= \begin{cases}1, & \text { if } \frac{|S[l, k]|^{2}}{|D[l, k]|^{2}}>1 \\ 0, & \text { otherwise, }\end{cases}
$$

where the threshold used here is equivalent to $0 \mathrm{~dB}$ (Wang, 2005). The IRM is computed as

$$
t[l, k]=\operatorname{IRM}[l, k]=\sqrt{\frac{|S[l, k]|^{2}}{|S[l, k]|^{2}+|D[l, k]|^{2}}} .
$$

The IBM and IRM are applied elementwise to each TF component of the noisy speech MS, for example:

$$
|\hat{S}[l, k]|=|X[l, k]| \cdot \operatorname{IRM}[l, k] .
$$

\section{B. MS training targets}

Here, training targets derived from the clean speech MS are described. The magnitude or power of the clean speech spectrum has been found difficult for a DNN to learn (Wang et al., 2014; Xu et al., 2015). Hence, the clean speech MS must be compressed, which leads to the decibel value of the clean speech MS as a training target:

$$
t[l, k]=\left|S_{\mathrm{dB}}[l, k]\right|=20 \log _{10}(|S[l, k]|) .
$$

Standardising $\left|S_{\mathrm{dB}}[l, k]\right|$ has also been found beneficial (Xu et al., 2015): ${ }^{1}$

$$
t[l, k]=z\left(\left|S_{\mathrm{dB}}[l, k]\right|\right)=\frac{\left|S_{\mathrm{dB}}[l, k]\right|-\mu_{k}}{\sigma_{k}},
$$

where $\mu_{k}$ and $\sigma_{k}^{2}$ are the mean and variance of the $k^{t h}$ discrete-frequency bin, respectively. Min-max normalisation has also been found to facilitate training (Wang et al., 2014):

$$
t[l, k]=|S[l, k]|^{\prime}=\frac{|S[l, k]|-\min _{k}(|S[l, k]|)}{\max _{k}(|S[l, k]|)-\min _{k}(|S[l, k]|)},
$$

where $\min _{k}(\cdot)$ and $\max _{k}(\cdot)$ find the minimum and maximum values for the $k^{t h}$ discrete-frequency bin, respectively. Applying min-max normalisation to the decibel values also facilitates training (Wang et al., 2014), where $\left|S_{\mathrm{dB}}[l, k]\right|^{\prime}$ is formed by replacing $|S[l, k]|$ in Equation (16) with $\left|S_{\mathrm{dB}}[l, k]\right|$.

We also investigate the cumulative distribution function (CDF) of $\left|S_{\mathrm{dB}}[l, k]\right|$ as a compression function, as motivated by (Nicolson and Paliwal, 2019a). The distribution of $\left|S_{\mathrm{dB}}[l, 64]\right|$ is shown in Figure 1 (a), which indicates that $\left|S_{\mathrm{dB}}[l, k]\right|$ follows a normal distribution. Hence, we assume that $\left|S_{\mathrm{dB}}[l, k]\right|$ follows a normal distribution: $\left|S_{\mathrm{dB}}[l, k]\right| \sim \mathcal{N}\left(\mu_{k}, \sigma_{k}^{2}\right)$, where $\mu_{k}$ and $\sigma_{k}^{2}$ are the mean and variance of the $k^{t h}$ discrete-frequency bin, respectively. The normal CDF of $\left|S_{\mathrm{dB}}[l, k]\right|$ is given by

$$
t[l, k]=F\left(\left|S_{\mathrm{dB}}[l, k]\right|\right)=\frac{1}{2}\left[1+\operatorname{erf}\left(\frac{\left|S_{\mathrm{dB}}[l, k]\right|-\mu_{k}}{\sigma_{k} \sqrt{2}}\right)\right],
$$

where $\operatorname{erf}(\cdot)$ is the error function.
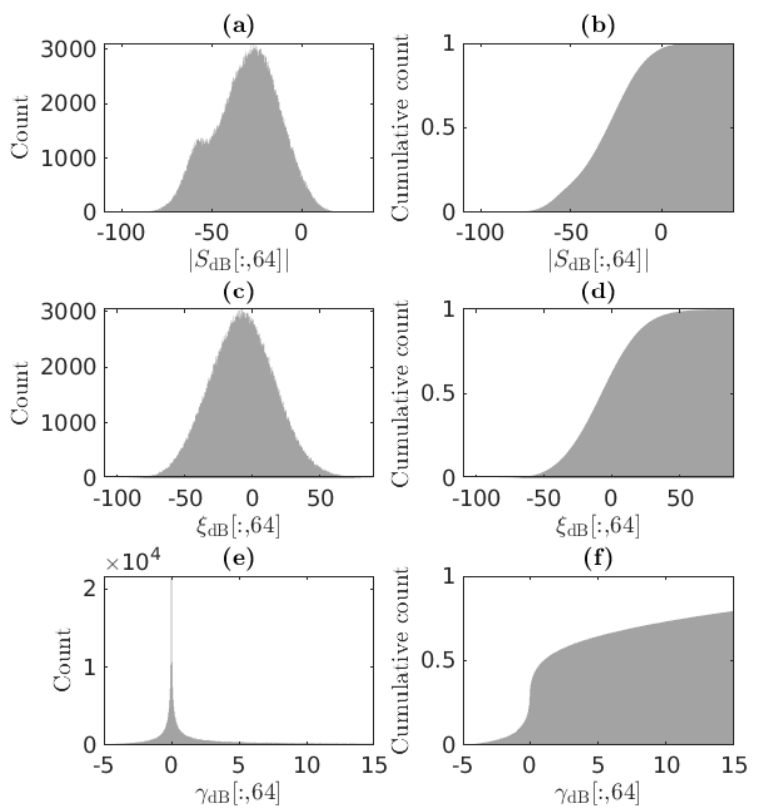

FIG. 1. The histogram of (a) $\left|S_{\mathrm{dB}}[l, 64]\right|$, (c) $\xi_{\mathrm{dB}}[l, 64]$, and (e) $\gamma_{\mathrm{dB}}[l, 64]$, and the normalised cumulative histogram of (b) $\left|S_{\mathrm{dB}}[l, 64]\right|,(\mathrm{d}) \xi_{\mathrm{dB}}[l, 64]$, and (f) $\gamma_{\mathrm{dB}}[l, 64]$. The histograms are found over the sample described in Subsection VD.

\section{MMSE estimator training targets}

As in (Nicolson and Paliwal, 2019a), the instantaneous a priori SNR can be used as a training target. The instantaneous a priori SNR is computed by using the instantaneous values $|S[l, k]|^{2}$ and $|D[l, k]|^{2}$ in place 
of $\lambda_{s}[l, k]$ and $\lambda_{d}[l, k]$, respectively, in Equation (8). However, the power values of the instantaneous a priori SNR are difficult to train (Nicolson and Paliwal, 2019a). Thus, a compression function must be used to facilitate training. As with the clean speech MS, the decibel value of the instantaneous a priori SNR can be used as a training target:

$$
t[l, k]=\xi_{\mathrm{dB}}[l, k]=10 \log _{10}(\xi[l, k]) .
$$

An additional training target is formed by standardising $\xi_{\mathrm{dB}}[l, k]$, where $z\left(\xi_{\mathrm{dB}}[l, k]\right)$ is formed by replacing $\left|S_{\mathrm{dB}}[l, k]\right|$ in Equation (15) with $\xi_{\mathrm{dB}}[l, k]$. We also apply min-max normalisation to $\xi[l, k]$ and $\xi_{\mathrm{dB}}[l, k]$, where $\xi[l, k]^{\prime}$ and $\xi_{\mathrm{dB}}[l, k]^{\prime}$ are formed by replacing $|S[l, k]|$ in Equation (16) with $\xi[l, k]$ and $\xi_{\mathrm{dB}}[l, k]$, respectively.

In (Nicolson and Paliwal, 2019a), the cumulative distribution function (CDF) of $\xi_{\mathrm{dB}}[l, k]$ was used as the compression function, as part of the Deep Xi framework. ${ }^{2}$ It was assumed that $\xi_{\mathrm{dB}}[l, k]$ is distributed normally: $\xi_{\mathrm{dB}}[l, k] \sim \mathcal{N}\left(\mu_{k}, \sigma_{k}^{2}\right)$, where $\mu_{k}$ and $\sigma_{k}^{2}$ are the mean and variance of the $k^{\text {th }}$ discrete-frequency bin, respectively. The distribution of $\xi_{\mathrm{dB}}[l, 64]$ is shown in Figure 1 (c). The normal CDF of $\xi_{\mathrm{dB}}[l, k]$ is given by

$$
t[l, k]=F\left(\xi_{\mathrm{dB}}[l, k]\right)=\frac{1}{2}\left[1+\operatorname{erf}\left(\frac{\xi_{\mathrm{dB}}[l, k]-\mu_{k}}{\sigma_{k} \sqrt{2}}\right)\right] .
$$

For the Deep Xi framework, the maximum likelihood (ML) a posteriori SNR estimate is used with the MMSESTSA and MMSE-LSA estimators: $\hat{\gamma}[l, k]=\xi[l, k]+1$. Instead, we propose to jointly estimate both the a priori and a posteriori SNR. While the MMSE-STSA and MMSE-LSA estimators largely rely on the a priori SNR, some performance improvement may be realised by additionally estimating the a posteriori SNR (Cappe, 1994). The decibel value of the instantaneous a priori and $a$ posteriori SNR can be used as a training target:

$$
t[l, k]=\left[\xi_{\mathrm{dB}}[l, k] ; \gamma_{\mathrm{dB}}[l, k]\right],
$$

where $[\cdot ; \cdot]$ is the concatenation operation and $\gamma_{\mathrm{dB}}[l, k]$ is computed as per Equation (21).

$$
\gamma_{\mathrm{dB}}[l, k]=10 \log _{10}(\gamma[l, k]) .
$$

We also apply min-max normalisation to $[\xi[l, k] ; \gamma[l, k]]$ and $\left[\xi_{\mathrm{dB}}[l, k] ; \gamma_{\mathrm{dB}}[l, k]\right]$, giving $\left[\xi[l, k]^{\prime} ; \gamma[l, k]^{\prime}\right]$ and $\left[\xi_{\mathrm{dB}}[l, k]^{\prime} ; \gamma_{\mathrm{dB}}[l, k]^{\prime}\right]$, respectively. $\quad \gamma[l, k]^{\prime}$ and $\gamma_{\mathrm{dB}}[l, k]^{\prime}$ are formed by replacing $|S[l, k]|$ in Equation (16) with $\gamma[l, k]$ and $\gamma_{\mathrm{dB}}[l, k]$, respectively.

We also propose to use the cumulative distribution function $(\mathrm{CDF})$ of $\xi_{\mathrm{dB}}[l, k]$ and $\gamma_{\mathrm{dB}}[l, k]$ as a compression function to form a training target:

$$
t[l, k]=\left[F\left(\xi_{\mathrm{dB}}[l, k]\right) ; F\left(\gamma_{\mathrm{dB}}[l, k]\right)\right] .
$$

The distribution of $\gamma_{\mathrm{dB}}[l, 64]$ is shown in Figure 1 (e), which indicates that $\gamma_{\mathrm{dB}}[l, k]$ follows a super-Gaussian distribution. Hence, we assume that $\gamma_{\mathrm{dB}}[l, k]$ follows a
Laplace distribution: $\gamma_{\mathrm{dB}}[l, k] \mid \sim \operatorname{Laplace}\left(\zeta_{k}, b_{k}\right)$, where $\zeta_{k}$ and $b_{k}$ are the location and scale parameters of the $k^{t h}$ discrete-frequency bin, respectively. The Laplace CDF of $\gamma_{\mathrm{dB}}[l, k]$ is given by

$$
\begin{aligned}
F\left(\gamma_{\mathrm{dB}}[l, k]\right)= & \frac{1}{2}+\frac{1}{2} \operatorname{sgn}\left(\gamma_{\mathrm{dB}}[l, k]-\right. \\
& \left.\zeta_{k}\right)\left(1-\exp \left(\frac{\left|\gamma_{\mathrm{dB}}[l, k]-\zeta_{k}\right|}{b_{k}}\right)\right),
\end{aligned}
$$

where $\operatorname{sgn}(\cdot)$ is the sign function.

\section{EXPERIMENT SETUP}

\section{A. Deep neural network: ResNet TCN}

A modified version of the residual network (ResNet) TCN from (Zhang et al., 2020) is used to evaluate each training target. ${ }^{3}$ It is shown from input to output in Figure 2. Its input at time-frame $l$ is $\left|\mathbf{X}_{l}\right|=$ $\left\{|X[l, 0]|,|X[l, 1]|, \ldots,\left|X\left[l, N_{d} / 2\right]\right|\right\}$, which is the 257point single-sided noisy speech MS that includes the DC and Nyquist discrete-frequency bins. Its output at timeframe $l$ is $\hat{\mathbf{t}}_{l}=\left\{\hat{t}[l, 0], \hat{t}[l, 1], \ldots, \hat{t}\left[l, N_{d} / 2\right]\right\}$. The input is first transformed by $\mathbf{F C}$, a fully-connected layer of size $d_{\text {model }}=256$. Instead of applying layer normalisation (Ba et al., 2016) followed by the rectifier linear function to FC, as in (Zhang et al., 2020), we apply the rectifier linear activation function followed by layer normalisation without the scale and shift operations. This reduces overfitting, as demonstrated in (Xu et al., 2019). The FC layer is followed by $B=40$ bottleneck residual blocks, where $b=1,2, \ldots, B$ is the block index. Residual blocks are facilitated by adding the block's input to its output, preventing the vanishing and exploding gradient problems (He et al., 2016).

Each block contains three one-dimensional causal dilated convolutional units. Here, we modify the preactivation of the convolutional units in (Zhang et al., 2020) by using the rectifier linear activation function followed by layer normalisation without the scale and shift operations (again following (Xu et al., 2019)). The kernel size, output size, and dilation rate for each convolutional unit is denoted in Figure 2 as (kernel size, output size, dilation rate). The first and third convolutional units have a kernel size of 1 , whilst the second has a kernel size of $k=3$. The first and second convolutional units have an output size of $d_{b}=64$, whilst the third has an output size of $d_{\text {model }}$. The first and third convolutional units have a dilation rate of 1 , while the second employs a dilation rate of $d$, providing a receptive field over previous time steps. The dilation rate for the second convolutional unit, $d$, is cycled as the block index, $b$, increases: $d=2^{\left(b-1 \bmod \left(\log _{2}(D)+1\right)\right.}$, where $\bmod$ is the modulo operation, and $D=16$ is the maximum dilation rate. The last block is followed by a fully-connected output layer, $\mathbf{O}$.

For training, the Adam algorithm (Kingma and Ba, 2014) with default hyper-parameters is used for gradient descent optimisation. Gradients are clipped between 


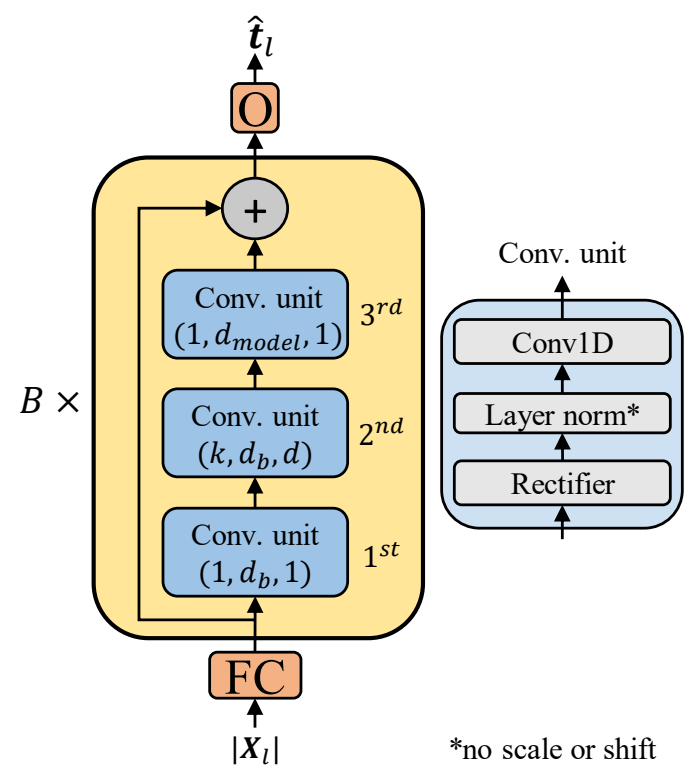

FIG. 2. (colour online) ResNet TCN. The kernel size, output size, and dilation rate for each convolutional unit is denoted as (kernel size, output size, dilation rate).

$[-1,1]$. A mini-batch size of 8 noisy speech signals is used for each training iteration. The noisy speech signals for each mini-batch are computed on the fly as follows: each clean speech recording selected for the mini-batch is mixed with a random section of a randomly selected noise recording at a randomly selected SNR level from the set specified for the training set. The selection order for the clean speech recordings is randomised for each epoch. The ResNet TCN is implemented in TensorFlow 2.3.0 (Abadi et al., 2015) and is available at https://github.com/anicolson/DeepXi.

\section{B. Output layer activation function and loss function}

In Table I, we specify the output layer activation function and the loss function used for each training target. For $\{t[l, k] \in \mathbb{R}: 0 \leq t[l, k] \leq 1\}$, the sigmoid activation function is applied to the output layer, and mean squared error (MSE) and binary cross-entropy (BCE) are both investigated as the loss function. For all other cases, no activation function is applied to the output layer and MSE is investigated as the loss function.

\section{Datasets}

Separate networks for each training target are trained and evaluated on the following datasets:
TABLE I. Range of values, output layer activation function, and loss function for each training target.

\begin{tabular}{|c|c|c|c|}
\hline Target, $t[l, k]$ & Range & Outp. activat. & Loss function \\
\hline $\operatorname{IBM}[l, k]$ & {$[0,1]$} & Sigmoid & MSE or BCE \\
\hline $\operatorname{IRM}[l, k]$ & {$[0,1]$} & Sigmoid & MSE or BCE \\
\hline$|S[l, k]|^{\prime}$ & {$[0,1]$} & Sigmoid & MSE or BCE \\
\hline$\left|S_{\mathrm{dB}}[l, k]\right|$ & {$[-\infty, \infty]$} & Linear & MSE \\
\hline$z\left(\left|S_{\mathrm{dB}}[l, k]\right|\right)$ & {$[-\infty, \infty]$} & Linear & MSE \\
\hline$\left|S_{\mathrm{dB}}[l, k]\right|^{\prime}$ & {$[0,1]$} & Sigmoid & MSE \\
\hline$F\left(\left|S_{\mathrm{dB}}[l, k]\right|\right)$ & {$[0,1]$} & Sigmoid & MSE or BCE \\
\hline$\xi[l, k]^{\prime}$ & {$[0,1]$} & Sigmoid & MSE or BCE \\
\hline$\xi_{\mathrm{dB}}[l, k]$ & {$[-\infty, \infty]$} & Linear & MSE \\
\hline$z\left(\xi_{\mathrm{dB}}[l, k]\right)$ & {$[-\infty, \infty]$} & Linear & MSE \\
\hline$\xi_{\mathrm{dB}}[l, k]^{\prime}$ & {$[0,1]$} & Sigmoid & MSE or BCE \\
\hline$F\left(\xi_{\mathrm{dB}}[l, k]\right)$ & {$[0,1]$} & Sigmoid & MSE or BCE \\
\hline$\left[\xi[l, k]^{\prime} ; \gamma[l, k]^{\prime}\right]$ & {$[0,1]$} & Sigmoid & MSE or BCE \\
\hline$\left[\xi_{\mathrm{dB}}[l, k] ; \gamma_{\mathrm{dB}}[l, k]\right]$ & {$[-\infty, \infty]$} & Linear & MSE \\
\hline$\left[\xi_{\mathrm{dB}}[l, k]^{\prime} ; \gamma_{\mathrm{dB}}[l, k]^{\prime}\right]$ & {$[0,1]$} & Sigmoid & MSE or BCE \\
\hline$\left[F\left(\xi_{\mathrm{dB}}[l, k]\right) ; F\left(\gamma_{\mathrm{dB}}[l, k]\right)\right]$ & {$[0,1]$} & Sigmoid & MSE or BCE \\
\hline
\end{tabular}

\section{DEMAND Voice Bank dataset}

The DEMAND Voice Bank dataset (ValentiniBotinhao et al., 2016) has been used frequently as of late to evaluate deep learning approaches to speech enhancement/separation (Nikzad et al., 2020). The training set includes clean speech recordings from 28 speakers of the Voice Bank corpus (Veaux et al., 2013) (11572 recordings). It also includes two synthetic noise sources (speechshaped noise and babble, as described in (ValentiniBotinhao et al., 2016)), as well as eight real-world noise recordings from the DEMAND dataset (Thiemann et al., 2013). The clean speech and noise recordings are downsampled from $48 \mathrm{kHz}$ to $16 \mathrm{kHz}$. The noisy speech signals for the training set are formed by mixing each clean speech recording with a random section of a randomly selected noise recording at a random SNR level from the set $\{0,5,10,15\}(\mathrm{dB})$. This creates a set of 11572 noisy speech signals for training. The test set includes 824 clean speech recordings of two speakers from the Voice Bank corpus - 393 from $p 232$ and 431 from $p 257$ (Veaux et al., 2013). Both speakers are separate from those selected for the training set. A total of 20 different conditions are used to create the noisy speech, including five noise sources from the DEMAND dataset (separate from those included in the training set), and 4 SNR levels: $\{2.5,7.5,12.5,17.5\}(\mathrm{dB})$. The clean speech and noise recordings are downsampled from $48 \mathrm{kHz}$ to $16 \mathrm{kHz}$ prior to mixing. Approximately 20 different sentences for each speaker are used per condition, with the total number of noisy speech signals in the test set amounting to 824 .

\section{Deep Xi dataset}

The Deep Xi dataset (Nicolson, 2020) is larger than the DEMAND Voice Bank dataset, with a wider range of conditions for training. The test set also allows for the evaluation of individual noise sources and SNR lev- 
els. For the training and validation sets, 69708 clean speech recordings from the (Veaux et al., 2017) (41169 recordings, speakers $p 232$ and $p 257$ are excluded) and the train-clean-100 set of the Librispeech corpus (Panayotov et al., 2015) (28539 recordings) are used. The minimum, average, and maximum duration of the recordings is 1.4, 12.3 , and 17.2, respectively, for the train-clean-100 set and 1.2, 3.6, and 15.1, respectively, for the CSTR VCTK corpus. For the training and validation sets, noise recordings from the QUT-NOISE dataset (Dean et al., 2010), the Nonspeech dataset (Hu and Wang, 2010), the RSG10 dataset (voice babble, F16, and factory welding are excluded as they are used for the test set) (Steeneken and Geurtsen, 1988), the Urban Sound dataset (street music recording no. 26270 is excluded as it is used for the test set) (Salamon et al., 2014), the Environmental Background Noise dataset (Saki et al., 2016), the noise set from the MUSAN corpus (Snyder et al., 2015), multiple FreeSound packs, ${ }^{4}$ and coloured noise recordings (with an $\alpha$ value ranging from -2 to 2 in increments of 0.25 ). Noise recordings that are over 30 seconds in length are split into 30 second or less segments. This gives a total of 17458 noise recordings, each of a length less than or equal to 30 seconds. All clean speech and noise recordings are single-channel, with a sampling frequency of 16 $\mathrm{kHz}$ (recordings with a higher sampling frequency are downsampled to $16 \mathrm{kHz}$ ). The SNR levels from the set $\{q \in \mathbb{Z} \mid-10 \leq q \leq 20\}(\mathrm{dB})$, where $q$ is the SNR level, are used for the training set. For the validation set, 1000 clean speech and noise recordings are randomly selected (without replacement) and removed from the aforementioned clean speech and noise sets. Each clean speech recording is paired with one of the noise recordings. The clean speech recording is then mixed with a random section of the noise recordings at a randomly selected SNR level from the set $\{q \in \mathbb{Z} \mid-10 \leq q \leq 20\}$ (dB). This forms 1000 noisy speech signals for the validation set.

For the test set, recordings of four real-world noise sources, including two non-stationary and two coloured, are included in the test set. The two real-world nonstationary noise sources include voice babble from the RSG-10 noise dataset (Steeneken and Geurtsen, 1988) and street music (recording no. 26270) from the Urban Sound dataset (Salamon et al., 2014). The two real-world coloured noise sources include F16 and factory welding from the RSG-10 noise dataset (Steeneken and Geurtsen, 1988). 10 clean speech recordings are randomly selected (without replacement) from the test-clean set of the Librispeech corpus for each of the four noise recordings. The clean speech recordings from the test-clean set have a duration of up to 34 seconds. All clean speech and noise recordings are single-channel, with a sampling frequency of $16 \mathrm{kHz}$ (recordings with a higher sampling frequency are downsampled to $16 \mathrm{kHz}$ ). To create the noisy speech, a random section of the noise recording is selected and mixed with the clean speech at the following SNR levels: $\{-5,0,5,10,15\}(\mathrm{dB})$. This creates a test set of 200 noisy speech signals.

\section{RESULTS AND DISCUSSION}

In this section, we compare the objective quality and intelligibility, and robust ASR performance of CASA,
J. Acoust. Soc.' Am. 28 September 2020 .

\section{Sample}

The statistics required for some of the compression functions described in Section IV $\left(\mu_{k}, \sigma_{k}, \min _{k}(\cdot)\right.$, $\max _{k}(\cdot), \zeta_{k}$, and $\left.b_{k}\right)$ are estimated from a sample of 1000 randomly selected training examples (statistics are found seperately for each training set). For the Laplace CDF in Equation (23), $\zeta_{k}$ is assumed to be zero and $b_{k}$ is estimated using values greater than $\zeta_{k}$ - as a larger proportion of $\gamma_{\mathrm{dB}}[l, k]$ values are in the right tail (see Figure $1)$.

\section{E. ASR system}

Project DeepSpeech is an ASR system trained solely on clean speech (i.e. no multi-condition training is used). ${ }^{5}$ It is an open source implementation of the Deep Speech ASR system (Hannun et al., 2014). It uses 26 mel-frequency cepstral coefficients (MFCCs) as its input. It is used to evaluate the objective intelligibility of the training targets. It is also used to evaluate the front-end performance of each training target for robust ASR.

\section{F. Objective quality and intelligibility measures}

The objective quality and intelligibility measures used in this study are described as follows:

- CSIG is a composite objective measure that provides a mean opinion score (MOS) from 1-5 of the signal distortion (Hu and Loizou, 2008).

- CBAK is a composite objective measure that provides a MOS from 1-5 of the background-noise intrusiveness (Hu and Loizou, 2008).

- COVL is a composite objective measure that provides a MOS from $1-5$ of the overall signal quality (Hu and Loizou, 2008).

- PESQ is used for objective quality evaluation, and gives a score from -0.5-4.5 (Rix et al., 2001).

- STOI is used for objective intelligibility evaluation (Taal et al., 2010; Taal et al., 2011). The intelligibility score is given as a fraction from $0-1$ or as a percentage.

- Segmental SNR (SegSNR) is used as an objective quality measure (Mermelstein, 1979).

- WER is used as an objective measure of intelligibility. The WER is calculated by

$$
\mathrm{WER}=100 \frac{\mathcal{D}(H, R)}{N},
$$

where $H$ is the hypothesis transcript, $R$ is the reference transcript, and $N$ number of words in $R$. $\mathcal{D}(H, R)$ is the Levenshtein distance between $H$, and $R$. The WER is given as a fraction from $0-1$ or as a percentage.

MS, and MMSE training targets, as well as compression 
TABLE II. Mean objective scores on the test set of the DEMAND Voice Bank dataset described in Subsection V C 1. The highest score for each measure - except WER - is in boldface. The lowest WER is in boldface.

\begin{tabular}{|c|c|c|c|c|c|c|c|c|c|c|}
\hline Category & Target & Loss & MMSE est. & CSIG & CBAK & COVL & PESQ & SegSNR & STOI $(\%)$ & WER (\%) \\
\hline- & Noisy speech & - & - & 3.50 & 2.47 & 2.73 & 1.99 & 1.71 & 91.53 & 30.03 \\
\hline \multirow{4}{*}{ 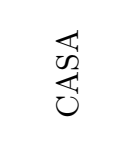 } & IBM & MSE & - & 1.99 & 2.96 & 1.96 & 2.11 & 9.11 & 92.97 & 36.60 \\
\hline & IBM & $\mathrm{BCE}$ & - & 2.12 & 2.96 & 2.03 & 2.13 & 9.04 & 92.73 & 36.87 \\
\hline & IRM & MSE & - & 4.17 & 3.29 & 3.46 & 2.73 & 8.04 & 93.74 & 25.52 \\
\hline & IRM & $\mathrm{BCE}$ & - & 4.16 & 3.25 & 3.45 & 2.72 & 7.55 & 93.87 & 25.57 \\
\hline \multirow{7}{*}{$\sum_{\Sigma}^{\pi \Omega}$} & $|S|^{\prime}$ & $\mathrm{BCE}$ & - & 4.14 & 3.20 & 3.44 & 2.72 & 6.64 & 93.18 & 26.92 \\
\hline & $\left|S_{\mathrm{dB}}\right|$ & MSE & - & 4.11 & 3.06 & 3.40 & 2.68 & 4.80 & 91.16 & 28.31 \\
\hline & $z\left(\left|S_{\mathrm{dB}}\right|\right)$ & MSE & - & 4.07 & 3.05 & 3.38 & 2.70 & 4.69 & 90.99 & 29.59 \\
\hline & $\left|S_{\mathrm{dB}}\right|^{\prime}$ & MSE & - & 4.02 & 2.94 & 3.27 & 2.51 & 4.30 & 91.15 & 28.88 \\
\hline & $\left|S_{\mathrm{dB}}\right|^{\prime}$ & $\mathrm{BCE}$ & - & 4.09 & 3.08 & 3.38 & 2.67 & 5.22 & 91.88 & 28.04 \\
\hline & $F\left(\left|S_{\mathrm{dB}}\right|\right)$ & MSE & - & 3.71 & 2.69 & 2.91 & 2.13 & 3.38 & 89.46 & 32.75 \\
\hline & $F\left(\left|S_{\mathrm{dB}}\right|\right)$ & $\mathrm{BCE}$ & - & 3.91 & 2.92 & 3.18 & 2.45 & 4.45 & 90.19 & 30.77 \\
\hline \multirow{22}{*}{ 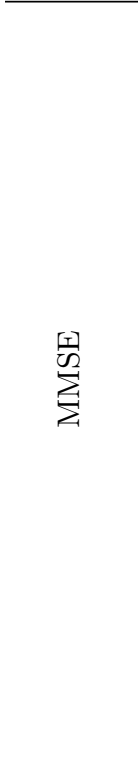 } & $\xi^{\prime}$ & MSE & MMSE-LSA & 3.51 & 2.49 & 2.75 & 2.03 & 1.98 & 91.86 & 58.85 \\
\hline & $\xi^{\prime}$ & MSE & SRWF & 3.51 & 2.48 & 2.74 & 2.02 & 1.87 & 91.80 & 57.91 \\
\hline & $\xi^{\prime}$ & $\mathrm{BCE}$ & MMSE-LSA & 3.50 & 2.48 & 2.73 & 2.02 & 1.99 & 91.81 & 29.32 \\
\hline & $\xi^{\prime}$ & $\mathrm{BCE}$ & SRWF & 3.49 & 2.47 & 2.73 & 2.01 & 1.87 & 91.76 & 29.38 \\
\hline & $\xi_{\mathrm{dB}}$ & MSE & MMSE-LSA & 4.19 & 3.40 & 3.52 & 2.83 & 9.03 & 93.31 & 25.78 \\
\hline & $\xi_{\mathrm{dB}}$ & MSE & SRWF & 4.18 & 3.32 & 3.48 & 2.76 & 8.38 & 93.36 & 26.16 \\
\hline & $z\left(\xi_{\mathrm{dB}}\right)$ & MSE & MMSE-LSA & 4.20 & 3.36 & 3.52 & 2.81 & 8.54 & 93.64 & 25.74 \\
\hline & $z\left(\xi_{\mathrm{dB}}\right)$ & MSE & SRWF & 4.16 & 3.25 & 3.45 & 2.71 & 7.68 & 93.66 & 26.14 \\
\hline & $\xi_{\mathrm{dB}}^{\prime}$ & MSE & MMSE-LSA & 4.21 & 3.36 & 3.54 & 2.83 & 8.40 & 93.37 & 26.75 \\
\hline & $\xi_{\mathrm{dB}}^{\prime}$ & MSE & SRWF & 4.18 & 3.25 & 3.47 & 2.73 & 7.47 & 93.41 & 26.87 \\
\hline & $\xi_{\mathrm{dB}}^{\prime}$ & $\mathrm{BCE}$ & MMSE-LSA & 4.09 & 3.34 & 3.40 & 2.70 & 9.15 & 93.34 & 26.52 \\
\hline & $\xi_{\mathrm{dB}}^{\prime}$ & $\mathrm{BCE}$ & SRWF & 4.06 & 3.26 & 3.34 & 2.61 & 8.55 & 93.34 & 26.89 \\
\hline & $F\left(\xi_{\mathrm{dB}}\right)$ & MSE & MMSE-LSA & 4.21 & 3.40 & 3.55 & 2.86 & 8.84 & 93.53 & 25.83 \\
\hline & $F\left(\xi_{\mathrm{dB}}\right)$ & MSE & SRWF & 4.18 & 3.31 & 3.49 & 2.77 & 8.12 & 93.60 & 26.00 \\
\hline & $F\left(\xi_{\mathrm{dB}}\right)$ & $\mathrm{BCE}$ & MMSE-LSA & 4.24 & 3.42 & 3.57 & 2.87 & 9.04 & 93.61 & 25.97 \\
\hline & $F\left(\xi_{\mathrm{dB}}\right)$ & $\mathrm{BCE}$ & SRWF & 4.21 & 3.34 & 3.51 & 2.79 & 8.37 & 93.66 & 25.99 \\
\hline & {$\left[\xi^{\prime} ; \gamma^{\prime}\right]$} & $\mathrm{BCE}$ & MMSE-LSA & 3.51 & 2.49 & 2.74 & 2.01 & 1.96 & 91.72 & 29.71 \\
\hline & {$\left[\xi_{\mathrm{dB}} ; \gamma_{\mathrm{dB}}\right]$} & MSE & MMSE-LSA & 4.19 & 3.42 & 3.52 & 2.83 & 9.22 & 93.64 & 26.01 \\
\hline & $\xi_{\mathrm{dB}}^{\prime} ; \gamma_{\mathrm{dB}}^{\prime}$ & MSE & MMSE-LSA & 4.18 & 3.37 & 3.48 & 2.76 & 9.14 & 93.41 & 27.30 \\
\hline & {$\left[\xi_{\mathrm{dB}}^{\prime} ; \gamma_{\mathrm{dB}}^{\prime}\right]$} & $\mathrm{BCE}$ & MMSE-LSA & 4.20 & 3.39 & 3.53 & 2.82 & 8.84 & 93.24 & 26.53 \\
\hline & {$\left[F\left(\xi_{\mathrm{dB}}\right) ; F\left(\gamma_{\mathrm{dB}}\right)\right]$} & MSE & MMSE-LSA & 4.22 & 3.38 & 3.55 & 2.85 & 8.47 & 93.65 & 25.99 \\
\hline & {$\left[F\left(\xi_{\mathrm{dB}}\right) ; F\left(\gamma_{\mathrm{dB}}\right)\right]$} & $\mathrm{BCE}$ & MMSE-LSA & 4.26 & 3.41 & 3.59 & 2.88 & 8.75 & 93.50 & 26.57 \\
\hline
\end{tabular}

and loss functions. The training targets are first evaluated on the DEMAND Voice Bank dataset, followed by the Deep Xi dataset. Epoch 125 and 150 for the DEMAND Voice Bank and Deep Xi datasets were used for evaluation, respectively. The TCNs required more epochs for the validation loss to converge on the Deep Xi dataset. The time-frame index, $l$, and the discrete-frequency bin, $k$, are omitted from the notation henceforth, for convenience. The SMM from (Williamson et al., 2016) was not included in the evaluation as repeated training runs would not converge (for clip values in the range 1.0-15.0). Moreover, $|S|^{\prime}+\mathrm{MSE}$ and $\left[\xi^{\prime} ; \gamma^{\prime}\right]+\mathrm{MSE}$ would not converge after repeated training runs. For difference testing, the two-sample $t$-test $(\alpha=0.05)$ is used henceforth, unless otherwise specified.

\section{A. DEMAND Voice Bank dataset objective scores}

The mean objective scores for each training target on the DEMAND Voice Bank dataset are given in Ta- ble II. First, we compare the objective quality and intelligibility scores of the three training target categories. CASA training targets, in particular the IRM, produced the most intelligible enhanced/separated speech. A significant difference between the STOI and WER scores of the IRM and all MS training targets exists (except for WER between IRM and $|S|^{\prime}$, and IRM and $\left|S_{\mathrm{dB}}\right|^{\prime}+\mathrm{BCE}$ ). However, this is not the case between the STOI and WER scores of the IRM and the MMSE training targets (except for $\xi^{\prime}$ and $\left.\left[\xi^{\prime} ; \gamma^{\prime}\right]\right)$. The MMSE training targets produced enhanced/separated speech with the highest quality. A significant difference between the objective scores of $\left[F\left(\xi_{\mathrm{dB}}\right) ; F\left(\gamma_{\mathrm{dB}}\right)\right]$ and $F\left(\xi_{\mathrm{dB}}\right)$, and all CASA and MS training targets exists (for CSIG, CBAK, COVL, and PESQ, and for the MMSE-LSA estimator). When comparing the objective quality scores of CASA and MS training targets, a significant difference between the IRM and $|S|^{\prime},\left|S_{\mathrm{dB}}\right|^{\prime}+\mathrm{MSE}$, and $F\left(\left|S_{\mathrm{dB}}\right|\right)$ exists (for CSIG, CBAK, COVL, PESQ, and SegSNR). However, this is not the case for the objective quality scores of the 
IRM compared to the remaining MS training targets (for CSIG, CBAK, COVL, PESQ, and SegSNR).

Next, we provide a further analysis of the CASA training targets. For the IBM, there is only a significant difference between the MSE and BCE loss functions for CSIG. For the IRM, there is only a significant difference between the MSE and BCE loss functions for SegSNR. This indicates that the choice of loss function has little impact on the performance of CASA training targets. The IBM is able to produce significantly higher STOI and SegSNR scores than all MS training targets, except for $|S|^{\prime}$. The IBM's effectiveness at noise suppression and producing intelligible enhanced/separated speech has been observed previously (Wang et al., 2014). However, its main weakness lies in its inability to produce enhanced/separated speech at a high quality. As for the IRM, it was found to be the most suitable training target as a front-end for robust ASR, as it attained the highest STOI score and the lowest WER.

For the MS training targets, multiple compression functions were investigated. Min-max normalisation of the clean speech MS was found to be best, with $|S|^{\prime}$ producing the highest objective scores - significantly outperforming $z\left(\left|S_{\mathrm{dB}}\right|\right)$ and $F\left(\left|S_{\mathrm{dB}}\right|\right)$ (for all measures). For $\left|S_{\mathrm{dB}}\right|^{\prime}$, a significant difference was found between the objectives scores of each loss function (for all measures except WER), with BCE performing best. This was also the case for $F\left(\left|S_{\mathrm{dB}}\right|\right)$. This indicates that the choice of loss function has an impact on the performance of MS training targets.

Amongst the MMSE training targets, min-max normalisation of power values (not decibel values) produced the worst scores - where a significant difference between the objective scores of $\xi^{\prime}$ and $\left[\xi^{\prime} ; \gamma^{\prime}\right]$ and the other MMSE training targets exists (for all measures except WER). This is unexpected, as min-max normalisation of the clean speech MS performed best amongst the MS training targets. The best performing training target for $a$ priori SNR estimation was $F\left(\xi_{\mathrm{dB}}\right)$, producing the best CSIG, CBAK, COVL, PESQ, and STOI scores $\left(z\left(\xi_{\mathrm{dB}}\right)\right.$ produced the lowest WER and equaled the highest STOI score, and $\xi_{\mathrm{dB}}^{\prime}$ produced the highest SegSNR). The best performing training target for the joint estimation of the

\section{B. Deep Xi dataset objective scores}

In this subsection, we first evaluate the mean objective scores of the training targets on the Deep Xi dataset. We then evaluate the objective scores of the training targets on individual conditions, including multiple noise sources and SNR levels. Due to their lack of performance on the DEMAND Voice Bank dataset, we exclude $\xi^{\prime}$ and $\left[\xi^{\prime} ; \gamma^{\prime}\right]$ from this subsection.

The mean objective quality and intelligibility scores for each training target on the Deep Xi dataset are given in Table III. First, we compare the CASA, MS, and MMSE training targets to each other. Min-max normalisation of the clean speech MS produced the highest STOI score amongst all training targets, outperforming the IRM - which attained the highest STOI score for the DEMAND Voice Bank dataset. However, the a priori and a posteriori SNR was $\left[F\left(\xi_{\mathrm{dB}}\right) ; F\left(\gamma_{\mathrm{dB}}\right)\right]$, producing the best CSIG, COVL, PESQ, STOI, and WER scores $\left(\left[\xi_{\mathrm{dB}} ; \gamma_{\mathrm{dB}}\right]\right.$ produced the highest CBAK and SegSNR score). While $\left[F\left(\xi_{\mathrm{dB}}\right) ; F\left(\gamma_{\mathrm{dB}}\right)\right]$ produced higher CSIG, COVL, PESQ, STOI and SegSNR scores than $F\left(\xi_{\mathrm{dB}}\right)$ (for the MMSE-LSA estimator), a significant difference does not exist between any of the objective scores (for all measures, except between $F\left(\xi_{\mathrm{dB}}\right)+\mathrm{BCE}$ and $\left[F\left(\xi_{\mathrm{dB}}\right) ; F\left(\gamma_{\mathrm{dB}}\right)\right]+$ MSE for SegSNR). For $\xi^{\prime}$ and $\xi_{\mathrm{dB}}^{\prime}$, MSE produced higher objective scores than BCE for most measures. For $F\left(\xi_{\mathrm{dB}}\right),\left[\xi_{\mathrm{dB}}^{\prime} ; \gamma_{\mathrm{dB}}^{\prime}\right]$, and $\left[F\left(\xi_{\mathrm{dB}}\right) ; F\left(\gamma_{\mathrm{dB}}\right)\right]$, $\mathrm{BCE}$ produced higher objective scores for most measures. There is no significant difference between the objective scores between the MSE and BCE loss functions for the MMSE training targets (except for the CBAK and STOI scores between $\xi_{\mathrm{dB}}^{\prime}+\mathrm{MSE}$ and $\xi_{\mathrm{dB}}^{\prime}+\mathrm{BCE}$, and the WER between $\xi^{\prime}+\mathrm{MSE}$ and $\left.\xi^{\prime}+\mathrm{BCE}\right)$. The preceding points indicate that the choice of loss function for MMSE training targets is dependant upon the used compression function.

Between the MMSE estimators, the MMSE-LSA estimator produced the highest CSIG, CBAK, COVL, PESQ, SegSNR, and WER scores for most cases. The SRWF produced the highest STOI scores for most cases - at the cost of objective quality performance. The MMSE-LSA estimator outperformed the SRWF as a front-end for robust ASR, which is unexpected due to the higher STOI scores of the SRWF. There is also a significant difference between the CBAK, PESQ, and SegSNR scores, indicating that the MMSE-LSA estimator is better able to perform noise suppression. Next, we compare the objective scores of the SRWF to to the IRM, as they have the same form. $F\left(\xi_{\mathrm{dB}}\right)+\mathrm{BCE}+\mathrm{SRWF}$ produced higher CSIG, CBAK, COVL, PESQ, and SegSNR scores, while both IRM+MSE and IRM+BCE produce higher STOI and WER scores. There is a significant difference between the CBAK, COVL, PESQ, and SegSNR scores of $F\left(\xi_{\mathrm{dB}}\right)+\mathrm{BCE}+\mathrm{SRWF}$ and IRM+MSE. There is only a significant difference between the SegSNR of $F\left(\xi_{\mathrm{dB}}\right)+\mathrm{BCE}+\mathrm{SRWF}$ and IRM+BCE. The preceding points indicate that the IRM is biased towards intelligibility, rather than quality.

IRM produced a lower (better) WER than $|S|^{\prime}$, indicating that the STOI score for $|S|^{\prime}$ is anomalous. $|S|^{\prime}$ produced higher CSIG, COVL, and PESQ scores than IRM. However, there is no significant difference between the objective scores of $|S|^{\prime}$ and IRM for all measures. The MMSE training targets produced higher CSIG, CBAK, COVL, PESQ, and SegSNR scores, as well as a lower WER than the MS training targets. A significant difference between the CBAK and SegSNR scores of $|S|^{\prime}$ and $F\left(\xi_{\mathrm{dB}}\right)+\mathrm{MSE}+\mathrm{MMSE}-\mathrm{LSA}$ exists. $F\left(\xi_{\mathrm{dB}}\right)$ produced the highest CSIG, CBAK, COVL, and PESQ scores amongst all training targets, further indicating that MMSE training targets produce the highest objective quality scores. As with the results for the DEMAND Voice Bank dataset, the IRM produced a higher STOI score and a lower WER than the MMSE training targets. However, 
TABLE III. Mean objective scores on the test set of the Deep Xi dataset described in Subsection V C 2. The highest score for each measure - except WER - is in boldface. The lowest WER is in boldface.

\begin{tabular}{|c|c|c|c|c|c|c|c|c|c|c|}
\hline Category & Target & Loss & MMSE est. & CSIG & CBAK & COVL & PESQ & SegSNR & STOI (\%) & WER $(\%)$ \\
\hline- & Noisy speech & - & - & 2.26 & 1.80 & 1.67 & 1.24 & -0.25 & 77.75 & 58.88 \\
\hline 㭊 & $\begin{array}{l}\text { IBM } \\
\text { IBM } \\
\text { IRM } \\
\text { IRM }\end{array}$ & $\begin{array}{l}\text { MSE } \\
\text { BCE } \\
\text { MSE } \\
\text { BCE }\end{array}$ & $\begin{array}{l}- \\
- \\
- \\
-\end{array}$ & $\begin{array}{l}1.46 \\
1.42 \\
3.08 \\
3.11\end{array}$ & $\begin{array}{l}2.19 \\
2.19 \\
2.43 \\
2.43\end{array}$ & $\begin{array}{l}.34 \\
1.32 \\
2.36 \\
2.37\end{array}$ & $\begin{array}{l}1.34 \\
1.34 \\
1.73 \\
1.74\end{array}$ & $\begin{array}{l}5.37 \\
\mathbf{5 . 5 1} \\
4.16 \\
4.14\end{array}$ & $\begin{array}{l}80.58 \\
81.04 \\
85.68 \\
85.52\end{array}$ & $\begin{array}{l}56.87 \\
58.71 \\
\mathbf{4 1 . 5 8} \\
41.97\end{array}$ \\
\hline$\sum_{i}^{\pi / 2}$ & $\begin{array}{c}\mid S^{\prime} \\
\left|S_{\mathrm{dB}}\right| \\
z\left(\left|S_{\mathrm{dB}}\right|\right) \\
\left|S_{\mathrm{dB}}\right|^{\prime} \\
\left|S_{\mathrm{dB}}\right|^{\prime} \\
F\left(\left|S_{\mathrm{dB}}\right|\right) \\
F\left(\left|S_{\mathrm{dB}}\right|\right)\end{array}$ & $\begin{array}{l}\text { BCE } \\
\text { MSE } \\
\text { MSE } \\
\text { MSE } \\
\text { BCE } \\
\text { MSE } \\
\text { BCE }\end{array}$ & $\begin{array}{l}- \\
- \\
- \\
- \\
- \\
- \\
-\end{array}$ & $\begin{array}{l}3.14 \\
3.07 \\
2.83 \\
2.99 \\
2.99 \\
2.85 \\
2.95\end{array}$ & $\begin{array}{l}2.43 \\
2.36 \\
2.18 \\
2.30 \\
2.32 \\
2.15 \\
2.24\end{array}$ & $\begin{array}{l}2.41 \\
2.35 \\
2.14 \\
2.26 \\
2.27 \\
2.11 \\
2.23\end{array}$ & $\begin{array}{l}1.79 \\
1.72 \\
1.57 \\
1.64 \\
1.65 \\
1.47 \\
1.60\end{array}$ & $\begin{array}{l}3.66 \\
2.95 \\
1.95 \\
2.76 \\
3.02 \\
1.72 \\
2.12\end{array}$ & $\begin{array}{l}\mathbf{8 5 . 7 8} \\
82.38 \\
79.19 \\
81.44 \\
81.63 \\
80.58 \\
81.18\end{array}$ & $\begin{array}{l}43.10 \\
48.84 \\
55.89 \\
51.22 \\
50.43 \\
50.02 \\
50.61\end{array}$ \\
\hline 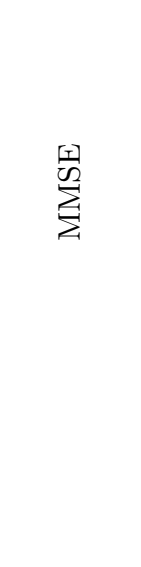 & $\begin{array}{c}\xi_{\mathrm{dB}} \\
\xi_{\mathrm{dB}} \\
z\left(\xi_{\mathrm{dB}}\right) \\
z\left(\xi_{\mathrm{dB}}\right) \\
\xi_{\mathrm{dB}}^{\prime} \\
\xi_{\mathrm{dB}}^{\prime} \\
\xi_{\mathrm{dB}}^{\prime} \\
\xi_{\mathrm{dB}}^{\prime} \\
F\left(\xi_{\mathrm{dB}}\right) \\
F\left(\xi_{\mathrm{dB}}\right) \\
F\left(\xi_{\mathrm{dB}}\right) \\
F\left(\xi_{\mathrm{dB}}\right) \\
{\left[\xi_{\mathrm{dB}} ; \gamma_{\mathrm{dB}}\right]} \\
{\left[\xi_{\mathrm{dB}}^{\prime} ; \gamma_{\mathrm{dB}}^{\prime}\right]} \\
{\left[\xi_{\mathrm{dB}}^{\prime} ; \gamma_{\mathrm{dB}}^{\prime}\right]} \\
{\left[F\left(\xi_{\mathrm{dB}}\right) ; F\left(\gamma_{\mathrm{dB}}\right)\right]} \\
\left.F\left(\xi_{\mathrm{dB}}\right) ; F\left(\gamma_{\mathrm{dB}}\right)\right]\end{array}$ & $\begin{array}{l}\text { MSE } \\
\text { MSE } \\
\text { MSE } \\
\text { MSE } \\
\text { MSE } \\
\text { MSE } \\
\text { BCE } \\
\text { BCE } \\
\text { MSE } \\
\text { MSE } \\
\text { BCE } \\
\text { BCE } \\
\text { MSE } \\
\text { MSE } \\
\text { BCE } \\
\text { MSE } \\
\text { BCE }\end{array}$ & $\begin{array}{c}\text { MMSE-LSA } \\
\text { SRWF } \\
\text { MMSE-LSA } \\
\text { SRWF } \\
\text { MMSE-LSA } \\
\text { SRWF } \\
\text { MMSE-LSA } \\
\text { SRWF } \\
\text { MMSE-LSA } \\
\text { SRWF } \\
\text { MMSE-LSA } \\
\text { SRWF } \\
\text { MMSE-LSA } \\
\text { MMSE-LSA } \\
\text { MMSE-LSA } \\
\text { MMSE-LSA } \\
\text { MMSE-LSA }\end{array}$ & $\begin{array}{l}3.07 \\
3.05 \\
3.06 \\
3.04 \\
3.02 \\
3.02 \\
2.87 \\
2.86 \\
\mathbf{3 . 1 6} \\
\mathbf{3 . 1 6} \\
3.15 \\
3.14 \\
3.08 \\
2.96 \\
2.94 \\
3.12 \\
3.11\end{array}$ & $\begin{array}{l}2.50 \\
2.45 \\
2.50 \\
2.47 \\
2.49 \\
2.45 \\
2.37 \\
2.34 \\
\mathbf{2 . 5 7} \\
2.54 \\
2.53 \\
2.49 \\
2.53 \\
2.39 \\
2.41 \\
\mathbf{2 . 5 7} \\
2.51\end{array}$ & $\begin{array}{l}2.38 \\
2.34 \\
2.37 \\
2.34 \\
2.34 \\
2.32 \\
2.21 \\
2.19 \\
\mathbf{2 . 4 7} \\
2.44 \\
2.46 \\
2.42 \\
2.39 \\
2.27 \\
2.27 \\
2.45 \\
2.41\end{array}$ & $\begin{array}{l}1.80 \\
1.75 \\
1.79 \\
1.74 \\
1.78 \\
1.74 \\
1.68 \\
1.65 \\
\mathbf{1 . 8 7} \\
1.83 \\
1.86 \\
1.81 \\
1.81 \\
1.70 \\
1.71 \\
\mathbf{1 . 8 7} \\
1.81\end{array}$ & $\begin{array}{l}4.99 \\
4.70 \\
5.12 \\
4.87 \\
4.95 \\
4.73 \\
4.48 \\
4.34 \\
5.24 \\
5.07 \\
4.84 \\
4.59 \\
5.27 \\
4.35 \\
4.56 \\
5.26 \\
4.87\end{array}$ & $\begin{array}{l}83.48 \\
83.68 \\
83.39 \\
83.60 \\
83.22 \\
83.48 \\
81.26 \\
81.60 \\
84.47 \\
84.76 \\
84.39 \\
84.62 \\
83.44 \\
82.77 \\
82.39 \\
84.26 \\
83.81\end{array}$ & $\begin{array}{l}45.10 \\
45.10 \\
45.51 \\
46.52 \\
45.35 \\
45.70 \\
49.50 \\
48.98 \\
43.23 \\
42.95 \\
43.68 \\
43.63 \\
45.65 \\
47.39 \\
47.76 \\
41.87 \\
43.90\end{array}$ \\
\hline
\end{tabular}

the MMSE training targets again produced higher objective quality scores than the IRM. When comparing the IRM and $F\left(\xi_{\mathrm{dB}}\right)+\mathrm{MSE}+\mathrm{MMSE}-\mathrm{LSA}$, a significant difference exists only between the CBAK, PESQ, and SegSNR scores. The IBM produced the highest SegSNR, outperforming $\left[\xi_{\mathrm{dB}} ; \gamma_{\mathrm{dB}}\right]$ and $\left[F\left(\xi_{\mathrm{dB}}\right) ; F\left(\gamma_{\mathrm{dB}}\right)\right]$. As a front-end for robust ASR, the IRM performed best, followed by the CDF compressed MMSE training targets, and then $|S|^{\prime}$. This order is the same as for the DEMAND Voice Bank dataset, indicating that the IRM is the most suitable training target as a front-end for robust ASR.

As with the DEMAND Voice Bank dataset, the choice off loss function appeared to have no impact on the performance of the CASA training targets (no significant difference exists between IBM+MSE and IBM+BCE, and IRM+MSE and IRM+BCE). For the MS training targets, $|S|^{\prime}$ again produced the highest objective scores. A significant difference between the objective scores of $|S|^{\prime}$ and all other MS training targets exists (except for the CSIG, CBAK, COVL, PESQ, and WER scores between $|S|^{\prime}$ and $\left.\left|S_{\mathrm{dB}}\right|\right)$. BCE again performed best for $\left|S_{\mathrm{dB}}\right|^{\prime}$ and $F\left(\left|S_{\mathrm{dB}}\right|\right)$ (a significant difference exists between the CBAK, COVL, and PESQ scores of $F\left(\left|S_{\mathrm{dB}}\right|\right)+\mathrm{MSE}$ and $\left.F\left(\left|S_{\mathrm{dB}}\right|\right)+\mathrm{BCE}\right)$, indicating that $\mathrm{BCE}$ is best for the MS training targets.
$F\left(\xi_{\mathrm{dB}}\right)$ performed best amongst the MMSE training targets, attaining the highest objective scores (except for SegSNR and WER). However, it cannot be claimed that $F\left(\xi_{\mathrm{dB}}\right)$ is superior to $\left[F\left(\xi_{\mathrm{dB}}\right) ; F\left(\gamma_{\mathrm{dB}}\right)\right]$, as there is no significant difference between their objective scores. As with the DEMAND Voice Bank dataset, the CDF was the best compression function for a priori SNR estimation and joint a priori and a posteriori SNR estimation. The MSE loss function performed best for $F\left(\xi_{\mathrm{dB}}\right)$ and $\left[F\left(\xi_{\mathrm{dB}}\right) ; F\left(\gamma_{\mathrm{dB}}\right)\right]$ (however, no significant difference exists), which is opposite to what was observed on the DEMAND Voice Bank dataset. As with the DEMAND Voice Bank dataset, the SRWF produced higher STOI scores and lower objective quality scores than the MMSELSA estimator (however, no significant difference exists between the objective scores of $F\left(\xi_{\mathrm{dB}}\right)+\mathrm{MSE}+\mathrm{MMSE}-$ LSA and $\left.F\left(\xi_{\mathrm{dB}}\right)+\mathrm{MSE}+\mathrm{SRWF}\right)$. The MMSE-LSA estimator again outperformed the SRWF as a front-end for robust ASR, further indicating that it is more suitable as a front-end than the SRWF.

\section{Objective scores on individual conditions}

As the mean objective score for each condition is computed from a low number of samples (10), we avoid difference testing henceforth. We also exclude PESQ and SegSNR scores to keep the following evaluation succinct. 
TABLE IV. Mean CSIG scores for each condition of the test set from the Deep Xi dataset described in Subsection V C 2 . The MMSE-LSA estimator is used by the MMSE training targets. The highest score for each condition is in boldface.

\begin{tabular}{|c|c|c|c|c|c|c|}
\hline \multirow{2}{*}{ Noise } & \multirow{2}{*}{ Target } & \multicolumn{5}{|c|}{ SNR } \\
\hline & & -5 & 0 & 5 & 10 & 15 \\
\hline \multirow{9}{*}{$\begin{array}{l}\frac{0}{0} \\
0 \\
0 \\
0 \\
0 \\
0 \\
0\end{array}$} & Noisy speech & 1.91 & 2.24 & 2.64 & 3.08 & 3.58 \\
\hline & IRM+MSE & 2.27 & 2.82 & 3.35 & 3.85 & \\
\hline & $\mathrm{IRM}+\mathrm{BCE}$ & 2.27 & 2.82 & 3.34 & 3.83 & 4.2 \\
\hline & $|S|^{\prime}+\mathrm{BCE}$ & 2.34 & 2.85 & 3.35 & 3.82 & 4.2 \\
\hline & $\left|S_{\mathrm{dB}}\right|+\mathrm{MSE}$ & 2.28 & 2.83 & 3.33 & 3.77 & 4.12 \\
\hline & $F\left(\xi_{\mathrm{dB}}\right)+\mathrm{MSE}$ & 2.25 & 2.82 & 3.36 & 3.88 & 4.35 \\
\hline & $F\left(\xi_{\mathrm{dB}}\right)+\mathrm{BCE}$ & 2.21 & 2.81 & 3.37 & 3.91 & 4.37 \\
\hline & {$\left[F\left(\xi_{\mathrm{dB}}\right) ; F\left(\gamma_{\mathrm{dB}}\right)\right]+\mathrm{MSE}$} & 2.22 & 2.78 & 3.34 & 3.85 & 4.34 \\
\hline & {$\left[F\left(\xi_{\mathrm{dB}}\right) ; F\left(\gamma_{\mathrm{dB}}\right)\right]+\mathrm{BCE}$} & 2.20 & 2.75 & 3.31 & 3.85 & 4.32 \\
\hline \multirow{8}{*}{ 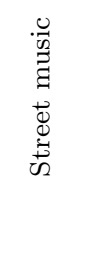 } & Noisy speech & 1.33 & & 1.97 & 2.41 & 2.93 \\
\hline & $\begin{array}{l}\text { IRM+MSE } \\
\text { IRM+BCE }\end{array}$ & $\begin{array}{l}1.85 \\
1.91\end{array}$ & $\begin{array}{l}2.33 \\
2.40\end{array}$ & $\begin{array}{l}2.82 \\
2.88\end{array}$ & $\begin{array}{l}3.29 \\
3.34\end{array}$ & $\begin{array}{l}3.75 \\
3.79\end{array}$ \\
\hline & $|S|^{\prime}+\mathrm{BCE}$ & 1.96 & 2.41 & 2.87 & 3.30 & 3.70 \\
\hline & $\left|S_{\mathrm{dB}}\right|+\mathrm{MSE}$ & 1.88 & 2.39 & 2.86 & 3.27 & 3.60 \\
\hline & $F\left(\xi_{\mathrm{dB}}\right)+\mathrm{MSE}$ & 1.91 & 2.44 & 2.95 & 3.46 & 3.9 \\
\hline & $F\left(\xi_{\mathrm{dB}}\right)+\mathrm{BCE}$ & 1.88 & 2.44 & 2.96 & 3.42 & 3.89 \\
\hline & {$\left[F\left(\xi_{\mathrm{dB}}\right) ; F\left(\gamma_{\mathrm{dB}}\right)\right]+\mathrm{MSE}$} & 1.87 & 2.39 & 2.92 & 3.43 & 3.94 \\
\hline & {$\left[F\left(\xi_{\mathrm{dB}}\right) ; F\left(\gamma_{\mathrm{dB}}\right)\right]+\mathrm{BCE}$} & 1.87 & 2.37 & 2.90 & 3.42 & 3.88 \\
\hline \multirow{9}{*}{$\begin{array}{l}\stackrel{0}{I} \\
\vec{I}\end{array}$} & Noisy speech & 1.24 & 1.58 & 1.99 & 2.46 & 2.98 \\
\hline & & 2.30 & 2.74 & 3.16 & & 4.04 \\
\hline & $\overline{\mathrm{IRM}}+\overline{\mathrm{BCE}}$ & 2.36 & 2.77 & 3.17 & 3.58 & 3.99 \\
\hline & $|S|^{\prime}+\mathrm{BCE}$ & 2.51 & 2.91 & 3.30 & 3.69 & 4.0 \\
\hline & $\left|S_{\mathrm{dB}}\right|+\mathrm{MSE}$ & 2.42 & 2.84 & 3.21 & 3.58 & 3.89 \\
\hline & $F\left(\xi_{\mathrm{dB}}\right)+\mathrm{MSE}$ & 2.40 & 2.83 & 3.24 & 3.72 & 4.14 \\
\hline & $F\left(\tilde{\xi}_{\mathrm{dB}}\right)+\mathrm{BCE}$ & 2.34 & 2.81 & 3.26 & 3.73 & 4.13 \\
\hline & $\left.F\left(\xi_{\mathrm{dB}}\right) ; F\left(\gamma_{\mathrm{dB}}\right)\right]+\mathrm{MSE}$ & 2.31 & 2.78 & 3.21 & 3.67 & 4.09 \\
\hline & {$\left[F\left(\xi_{\mathrm{dB}}\right) ; F\left(\gamma_{\mathrm{dB}}\right)\right]+\mathrm{BCE}$} & 2.28 & 2.74 & 3.20 & 3.68 & 4.14 \\
\hline \multirow{9}{*}{ 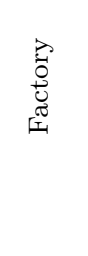 } & Noisy speech & 1.48 & 1.77 & 2.18 & 2.65 & 3.15 \\
\hline & IRM+MSE & 2.15 & 2.61 & 3.04 & 3.50 & 3.91 \\
\hline & $\overline{\mathrm{IRM}}+\mathrm{BCE}$ & 2.15 & 2.66 & 3.11 & 3.55 & 3.93 \\
\hline & $|S|^{\prime}+\mathrm{BCE}$ & 2.25 & 2.68 & 3.10 & 3.53 & 3.88 \\
\hline & $\left|S_{\mathrm{dB}}\right|+\mathrm{MSE}$ & 2.23 & 2.67 & 3.06 & 3.43 & 3.73 \\
\hline & $F\left(\xi_{\mathrm{dB}}\right)+\mathrm{MSE}$ & 2.20 & 2.70 & 3.16 & 3.60 & 3.94 \\
\hline & $F\left(\xi_{\mathrm{dB}}\right)+\mathrm{BCE}$ & 2.15 & 2.68 & 3.16 & 3.60 & 3.97 \\
\hline & {$\left[F\left(\xi_{\mathrm{dB}}\right) ; F\left(\gamma_{\mathrm{dB}}\right)\right]+\mathrm{MSE}$} & 2.10 & 2.63 & 3.10 & 3.54 & 3.95 \\
\hline & {$\left[F\left(\xi_{\mathrm{dB}}\right) ; F\left(\gamma_{\mathrm{dB}}\right)\right]+\mathrm{BCE}$} & 2.11 & 2.61 & 3.09 & 3.55 & 3.96 \\
\hline
\end{tabular}

Only the best performing training targets of each category from the previous subsections are included in the following evaluation. The MMSE-LSA estimator is used with the MMSE training targets.

The mean CSIG scores are given in Table VIB. $|S|^{\prime}$ attains the highest CSIG scores at lower SNR levels, while $F\left(\xi_{\mathrm{dB}}\right)$ attains the highest CSIG scores at higher SNR levels. This indicates that $|S|^{\prime}$ and $F\left(\xi_{\mathrm{dB}}\right)$ do not heavily distort the speech at lower and higher SNR levels, respectively. The noise source appears to have no significant impact on the best performing training target from condition to condition. The mean CBAK scores are given in Table VIB. $F\left(\xi_{\mathrm{dB}}\right)$ and $\left[F\left(\xi_{\mathrm{dB}}\right) ; F\left(\gamma_{\mathrm{dB}}\right)\right]$ attain the highest CBAK scores for most conditions. This demonstrates the noise suppression capabilities of MMSE training targets. $\left|S_{\mathrm{dB}}\right|$ attained the highest CBAK score for voice babble and F16 at an SNR level of $-5 \mathrm{~dB}$. The mean COVL scores are given in Table VIB $1 .|S|^{\prime}$ attains the highest COVL scores at lower SNR levels, while $F\left(\xi_{\mathrm{dB}}\right)$ attains the highest COVL scores at higher SNR levels. This indicates that $|S|^{\prime}$ and $F\left(\xi_{\mathrm{dB}}\right)$ produce high quality enhanced/separated speech at lower and higher
TABLE V. Mean CBAK scores for each condition of the test set from the Deep Xi dataset described in Subsection V C 2 . The MMSE-LSA estimator is used by the MMSE training targets. The highest score for each condition is in boldface.

\begin{tabular}{|c|c|c|c|c|c|c|}
\hline \multirow{2}{*}{ Noise } & \multirow{2}{*}{ Target } & \multicolumn{5}{|c|}{ SNR } \\
\hline & & -5 & 0 & 5 & 10 & 15 \\
\hline \multirow{9}{*}{$\begin{array}{l}\frac{0}{0} \\
\frac{0}{0} \\
0 \\
0 \\
0 \\
0 \\
0\end{array}$} & Noisy speech & 1.21 & 1.47 & 1.81 & 2.23 & 2.75 \\
\hline & IRM+MSE & 1.57 & 1.95 & 2.40 & 2.89 & 3.4 \\
\hline & $\mathrm{IRM}+\mathrm{BCE}$ & 1.59 & 1.97 & 2.41 & 2.89 & 3.3 \\
\hline & $|S|^{\prime}+\mathrm{BCE}$ & 1.66 & 2.02 & 2.43 & 2.87 & 3.2 \\
\hline & $\left|S_{\mathrm{dB}}\right|+\mathrm{MSE}$ & 1.72 & 2.04 & 2.39 & 2.75 & 3.05 \\
\hline & $F\left(\xi_{\mathrm{dB}}\right)+\mathrm{MSE}$ & 1.70 & 2.07 & 2.51 & 2.99 & 3.48 \\
\hline & $F\left(\xi_{\mathrm{dB}}\right)+\mathrm{BCE}$ & 1.60 & 2.00 & 2.47 & 2.98 & 3.49 \\
\hline & {$\left[F\left(\xi_{\mathrm{dB}}\right) ; F\left(\gamma_{\mathrm{dB}}\right)\right]+\mathrm{MSE}$} & 1.68 & 2.06 & 2.51 & 2.99 & 3.50 \\
\hline & {$\left[F\left(\xi_{\mathrm{dB}}\right) ; F\left(\gamma_{\mathrm{dB}}\right)\right]+\mathrm{BCE}$} & 1.64 & 1.97 & 2.42 & 2.94 & 3.45 \\
\hline \multirow{8}{*}{ 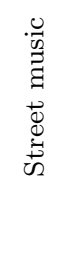 } & Noisy speech & & 1.36 & 1.70 & & \\
\hline & $\begin{array}{l}\text { IRM+MSE } \\
\text { IRM+BCE }\end{array}$ & $\begin{array}{l}1.63 \\
1.65\end{array}$ & $\begin{array}{l}1.97 \\
2.00\end{array}$ & $\begin{array}{l}2.36 \\
2.39\end{array}$ & $\begin{array}{l}2.79 \\
2.82\end{array}$ & $\begin{array}{l}3.24 \\
3.27\end{array}$ \\
\hline & $|S|^{\prime}+\mathrm{BCE}$ & 1.71 & $\begin{array}{l}2.00 \\
2.02\end{array}$ & 2.37 & 2.72 & $\begin{array}{l}3.21 \\
3.07\end{array}$ \\
\hline & $\left|S_{\mathrm{dB}}\right|+\mathrm{MSE}$ & 1.69 & 1.98 & 2.29 & 2.60 & 2.85 \\
\hline & $F\left(\xi_{\mathrm{dB}}\right)+\mathrm{MSE}$ & 1.75 & 2.11 & 2.51 & 2.97 & 3.42 \\
\hline & $F\left(\xi_{\mathrm{dB}}\right)+\mathrm{BCE}$ & 1.71 & 2.07 & 2.48 & 2.90 & 3.37 \\
\hline & $\left.F\left(\xi_{\mathrm{dB}}\right) ; F\left(\gamma_{\mathrm{dB}}\right)\right]+\mathrm{MSE}$ & 1.76 & 2.12 & 2.53 & 2.97 & 3.45 \\
\hline & $\left.F\left(\xi_{\mathrm{dB}}\right) ; F\left(\gamma_{\mathrm{dB}}\right)\right]+\mathrm{BCE}$ & 1.72 & 2.05 & 2.45 & 2.91 & 3.36 \\
\hline \multirow{9}{*}{$\stackrel{0}{\overrightarrow{1}}$} & Noisy speech & 1.13 & 1.37 & 1.67 & 2.04 & 2.49 \\
\hline & IRM & & 2.0 & 2.42 & 2.81 & \\
\hline & $\overline{\mathrm{IRM}}+\overline{\mathrm{BCE}}$ & 1.75 & 2.06 & 2.38 & 2.76 & 3.15 \\
\hline & $|S|^{\prime}+\mathrm{BCE}$ & 1.84 & 2.15 & 2.47 & 2.82 & 3.13 \\
\hline & $\left|S_{\mathrm{dB}}\right|+\mathrm{MSE}$ & 1.88 & 2.16 & 2.44 & 2.72 & 2.93 \\
\hline & $F\left(\xi_{\mathrm{dB}}\right)+\mathrm{MSE}$ & 1.87 & 2.21 & 2.58 & 2.98 & 3.35 \\
\hline & $F\left(\xi_{\mathrm{dB}}\right)+\mathrm{BCE}$ & 1.84 & 2.19 & 2.57 & 2.97 & 3.34 \\
\hline & $\left.F\left(\xi_{\mathrm{dB}}\right) ; F\left(\gamma_{\mathrm{dB}}\right)\right]+\mathrm{MSE}$ & 1.85 & 2.20 & 2.58 & 2.98 & 3.37 \\
\hline & $\left.F\left(\xi_{\mathrm{dB}}\right) ; F\left(\gamma_{\mathrm{dB}}\right)\right]+\mathrm{BCE}$ & 1.81 & 2.15 & 2.52 & 2.93 & 3.35 \\
\hline \multirow{8}{*}{ 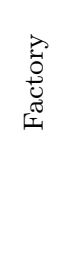 } & Noisy speech & 1.14 & 1.41 & 1.75 & 2.14 & 2.61 \\
\hline & MSE & & & 2.39 & 2.81 & 3.23 \\
\hline & $\begin{array}{l}\mathrm{IRM}+\mathrm{BC} \\
|S|^{\prime}+\mathrm{BC}\end{array}$ & $\begin{array}{l}1.67 \\
1.76\end{array}$ & $\begin{array}{l}2.03 \\
2.07\end{array}$ & $\begin{array}{l}2.41 \\
2.41\end{array}$ & 2.78 & $\begin{array}{l}3.24 \\
3.09\end{array}$ \\
\hline & $\left|S_{\mathrm{dB}}\right|+\mathrm{MSE}$ & 1.80 & 2.07 & 2.35 & 2.64 & 2.86 \\
\hline & $F\left(\xi_{\mathrm{dB}}\right)+\mathrm{MSE}$ & 1.83 & 2.19 & 2.57 & 2.97 & 3.32 \\
\hline & $F\left(\xi_{\mathrm{dB}}\right)+\mathrm{BCE}$ & 1.79 & 2.14 & 2.53 & 2.94 & 3.33 \\
\hline & $\left.F\left(\xi_{\mathrm{dB}}\right) ; F\left(\gamma_{\mathrm{dB}}\right)\right]+\mathrm{MSE}$ & 1.79 & 2.15 & 2.55 & 2.95 & 3.35 \\
\hline & {$\left[F\left(\xi_{\mathrm{dB}}\right) ; F\left(\gamma_{\mathrm{dB}}\right)\right]+\mathrm{BCE}$} & 1.78 & 2.10 & 2.49 & 2.90 & 3.30 \\
\hline
\end{tabular}

SNR levels, respectively. The mean CSIG, CBAK, and COVL scores from Tables VIB, VIB, and VIB 1 indicate that MS training targets produce the highest quality enhanced/separated speech at lower SNR levels, with the same being true for MMSE training targets at high SNR levels.

The mean STOI scores are given in Table VII. $|S|^{\prime}$ attains the highest STOI scores at lower SNR levels, while the IRM attains the highest STOI scores at higher SNR levels. This indicates that $|S|^{\prime}$ and the IRM produce enhanced/separated speech at the highest intelligibility at lower and higher SNR levels, respectively. The scores for WER are given in Table VII. It can be seen that there is no consistency between which training target performs best from condition to condition. Additionally, the STOI scores in Table VII are not indicative of the WER for each condition.

\section{CONCLUSION}

In this study, we compare CASA, MS, and MMSE training targets for clean speech MS estimation, with the 
TABLE VI. Mean COVL scores for each condition of the test set from the Deep Xi dataset described in Subsection V C 2. The MMSE-LSA estimator is used by the MMSE training targets. The highest score for each condition is in boldface.

\begin{tabular}{|c|c|c|c|c|c|c|}
\hline \multirow{2}{*}{ Noise } & \multirow{2}{*}{ Target } & \multicolumn{5}{|c|}{ SNR } \\
\hline & & -5 & 0 & 5 & 10 & 15 \\
\hline $\begin{array}{l}\frac{0}{0} \\
\frac{0}{0} \\
0 \\
0 \\
0 \\
0 \\
0\end{array}$ & $\begin{array}{c}\text { Noisy speech } \\
\text { IRM+MSE } \\
\text { IRM+BCE } \\
|S|^{\prime}+\mathrm{BCE} \\
\left|S_{\mathrm{dB}}\right|+\mathrm{MSE} \\
F\left(\xi_{\mathrm{dB}}\right)+\mathrm{MSE} \\
F\left(\xi_{\mathrm{dB}}\right)+\mathrm{BCE} \\
{\left[F\left(\xi_{\mathrm{dB}}\right) ; F\left(\gamma_{\mathrm{dB}}\right)\right]+\mathrm{MSE}} \\
{\left[F\left(\xi_{\mathrm{dB}}\right) ; F\left(\gamma_{\mathrm{dB}}\right)\right]+\mathrm{BCE}} \\
\end{array}$ & $\begin{array}{l}1.38 \\
1.61 \\
1.61 \\
\mathbf{1 . 6 6} \\
1.63 \\
1.61 \\
1.58 \\
1.58 \\
1.58\end{array}$ & $\begin{array}{l}1.58 \\
2.03 \\
2.03 \\
\mathbf{2 . 0 6} \\
\mathbf{2 . 0 6} \\
2.05 \\
2.04 \\
2.02 \\
1.98\end{array}$ & $\begin{array}{l}1.86 \\
2.50 \\
2.49 \\
2.52 \\
2.50 \\
2.55 \\
\mathbf{2 . 5 6} \\
2.54 \\
2.48\end{array}$ & $\begin{array}{l}2.23 \\
3.00 \\
2.98 \\
3.00 \\
2.94 \\
3.08 \\
\mathbf{3 . 1 0} \\
3.06 \\
3.03\end{array}$ & $\begin{array}{l}2.71 \\
3.49 \\
3.47 \\
3.44 \\
3.31 \\
3.58 \\
\mathbf{3 . 6 1} \\
3.60 \\
3.55\end{array}$ \\
\hline 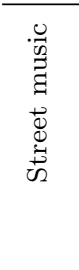 & $\begin{array}{c}\text { Noisy speech } \\
\text { IRM+MSE } \\
\text { IRM+BCE } \\
|S|^{\prime}+\mathrm{BCE} \\
\left|S_{\mathrm{dB}}\right|+\mathrm{MSE} \\
F\left(\xi_{\mathrm{dB}}\right)+\mathrm{MSE} \\
F\left(\xi_{\mathrm{dB}}\right)+\mathrm{BCE} \\
{\left[F\left(\xi_{\mathrm{dB}}\right) ; F\left(\gamma_{\mathrm{dB}}\right)\right]+\mathrm{MSE}} \\
{\left[F\left(\xi_{\mathrm{dB}}\right) ; F\left(\gamma_{\mathrm{dB}}\right)\right]+\mathrm{BCE}}\end{array}$ & $\begin{array}{l}1.07 \\
1.36 \\
1.39 \\
\mathbf{1 . 4 4} \\
1.39 \\
1.41 \\
1.39 \\
1.40 \\
1.38\end{array}$ & $\begin{array}{l}1.21 \\
1.71 \\
1.76 \\
1.78 \\
1.76 \\
\mathbf{1 . 8 2} \\
1.80 \\
1.81 \\
1.75\end{array}$ & $\begin{array}{l}1.44 \\
2.12 \\
2.17 \\
2.17 \\
2.16 \\
\mathbf{2 . 2 6} \\
2.25 \\
2.26 \\
2.19\end{array}$ & $\begin{array}{l}1.76 \\
2.57 \\
2.61 \\
2.59 \\
2.56 \\
\mathbf{2 . 7 7} \\
2.70 \\
2.76 \\
2.71\end{array}$ & $\begin{array}{l}2.21 \\
3.04 \\
3.07 \\
3.01 \\
2.90 \\
3.26 \\
3.20 \\
\mathbf{3 . 2 9} \\
3.18\end{array}$ \\
\hline $\begin{array}{l}0 \\
\vec{I}\end{array}$ & $\begin{array}{c}\text { Noisy speech } \\
\text { IRM+MSE } \\
\text { IRM+BCE } \\
|S|^{\prime}+\mathrm{BCE} \\
\left|S_{\mathrm{dB}}\right|+\mathrm{MSE} \\
F\left(\xi_{\mathrm{dB}}\right)+\mathrm{MSE} \\
F\left(\xi_{\mathrm{dB}}\right)+\mathrm{BCE} \\
{\left[F\left(\xi_{\mathrm{dB}}\right) ; F\left(\gamma_{\mathrm{dB}}\right)\right]+\mathrm{MSE}} \\
{\left[F\left(\xi_{\mathrm{dB}}\right) ; F\left(\gamma_{\mathrm{dB}}\right)\right]+\mathrm{BCE}}\end{array}$ & $\begin{array}{l}1.05 \\
1.66 \\
1.70 \\
\mathbf{1 . 8 3} \\
1.76 \\
1.74 \\
1.71 \\
1.70 \\
1.65\end{array}$ & $\begin{array}{l}1.20 \\
2.03 \\
2.05 \\
\mathbf{2 . 1 9} \\
2.12 \\
2.12 \\
2.12 \\
2.11 \\
2.05\end{array}$ & $\begin{array}{l}1.46 \\
2.42 \\
2.42 \\
2.57 \\
2.48 \\
2.55 \\
\mathbf{2 . 5 8} \\
2.54 \\
2.50\end{array}$ & $\begin{array}{l}1.80 \\
2.86 \\
2.84 \\
2.98 \\
2.87 \\
\mathbf{3 . 0 6} \\
\mathbf{3 . 0 6} \\
3.02 \\
3.01\end{array}$ & $\begin{array}{l}2.24 \\
3.31 \\
3.26 \\
3.36 \\
3.17 \\
\mathbf{3 . 4 9} \\
3.47 \\
3.46 \\
\mathbf{3 . 4 9}\end{array}$ \\
\hline 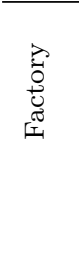 & $\begin{array}{c}\text { Noisy speech } \\
\text { IRM+MSE } \\
\text { IRM+BCE } \\
|S|^{\prime}+\mathrm{BCE} \\
\left|S_{\mathrm{dB}}\right|+\mathrm{MSE} \\
F\left(\xi_{\mathrm{dB}}\right)+\mathrm{MSE} \\
F\left(\xi_{\mathrm{dB}}\right)+\mathrm{BCE} \\
{\left[F\left(\xi_{\mathrm{dB}}\right) ; F\left(\gamma_{\mathrm{dB}}\right)\right]+\mathrm{MSE}} \\
{\left[F\left(\xi_{\mathrm{dB}}\right) ; F\left(\gamma_{\mathrm{dB}}\right)\right]+\mathrm{BCE}}\end{array}$ & $\begin{array}{l}1.17 \\
1.53 \\
1.52 \\
\mathbf{1 . 6 1} \\
1.60 \\
1.59 \\
1.56 \\
1.53 \\
1.52\end{array}$ & $\begin{array}{l}1.31 \\
1.87 \\
1.91 \\
1.94 \\
1.93 \\
\mathbf{1 . 9 9} \\
1.96 \\
1.93 \\
1.89\end{array}$ & $\begin{array}{l}1.55 \\
2.25 \\
2.30 \\
2.31 \\
2.28 \\
\mathbf{2 . 4 0} \\
\mathbf{2 . 4 0} \\
2.36 \\
2.31\end{array}$ & $\begin{array}{l}1.89 \\
2.69 \\
2.74 \\
2.74 \\
2.65 \\
\mathbf{2 . 8 5} \\
2.84 \\
2.79 \\
2.77\end{array}$ & $\begin{array}{l}2.31 \\
3.12 \\
3.13 \\
3.09 \\
2.95 \\
3.19 \\
\mathbf{3 . 2 2} \\
3.21 \\
3.20\end{array}$ \\
\hline
\end{tabular}

aim of determining which produces enhanced/separated speech at the highest quality and intelligibility, and also to determine which is most suitable as a front-end for robust ASR. CASA training targets, in particular the IRM, produced the highest objective intelligibility scores on the DEMAND Voice Bank dataset, and was the best front-end for robust ASR on the DEMAND Voice Bank and Deep Xi datasets. The IRM also produced higher objective quality scores than MS training targets on the DEMAND Voice Bank dataset. The choice of loss function had no impact on the performance of CASA training targets.

MS training targets produced the highest STOI score on the Deep Xi dataset. They also produced higher objective quality scores than CASA training targets on the Deep Xi dataset. Min-max normalisation of the clean speech MS found to be the best performing MS training target. When the values of the MS training target are in the range $[0,1], \mathrm{BCE}$ was found to perform best. MS training targets were also found to perform well at lower SNR levels ( -5 and $0 \mathrm{~dB})$. MMSE training targets produced the highest objective quality scores on the DE-
MAND Voice Bank and Deep Xi datasets. They also produced higher objective intelligibility scores on the DEMAND Voice Bank dataset, and performed better as a front-end for robust ASR on the DEMAND Voice Bank and Deep Xi datasets, than MS training targets. It was found that the CDF was the best compression function for a priori SNR and joint a priori and a posteriori SNR estimation. A significant difference between the objective scores produced by a priori SNR and joint a priori and a posteriori SNR estimation was not found.

Recommendations for future work include a comprehensive study on training targets that include the shorttime phase spectrum. Attention should also be payed to the variability of the objective scores produced by a training target over multiple training runs. Additionally, a dataset that contains more samples per condition would allow for a statistical analysis of the performance of the training targets over individual conditions.

TABLE VII. Mean STOI scores for each condition of the test set from the Deep Xi dataset described in Subsection V C 2. The MMSE-LSA estimator is used by the MMSE training targets. The highest score for each condition is in boldface.

\begin{tabular}{|c|c|c|c|c|c|c|}
\hline \multirow{2}{*}{ Noise } & \multirow{2}{*}{ Target } & \multicolumn{5}{|c|}{ SNR } \\
\hline & & -5 & 0 & 5 & 10 & 15 \\
\hline \multirow{9}{*}{$\begin{array}{l}\frac{0}{1} \\
0 \\
0 \\
0 \\
0 \\
0 \\
0 \\
0 \\
0\end{array}$} & Noisy speech & 57.77 & 70.62 & 81.84 & 89.85 & 94.65 \\
\hline & IRM+MSE & 65.56 & 79.65 & 88.73 & 94.00 & 96.68 \\
\hline & $\mathrm{IRM}+\mathrm{BCE}$ & 65.47 & 79.74 & 88.56 & 93.68 & 96.55 \\
\hline & $|S|^{\prime}+\mathrm{BCE}$ & 66.40 & 79.96 & 88.47 & 93.46 & 96.00 \\
\hline & $\left|S_{\mathrm{dB}}\right|+\mathrm{MSE}$ & 61.76 & 76.09 & 85.80 & 91.12 & 93.65 \\
\hline & $F\left(\xi_{\mathrm{dB}}\right)+\mathrm{MSE}$ & 61.98 & 77.84 & 88.02 & 93.52 & 96.53 \\
\hline & $F\left(\xi_{\mathrm{dB}}\right)+\mathrm{BCE}$ & 62.55 & 77.46 & 87.66 & 93.62 & 96.53 \\
\hline & $\left.F\left(\xi_{\mathrm{dB}}\right) ; F\left(\gamma_{\mathrm{dB}}\right)\right]+\mathrm{MSE}$ & 61.32 & 77.50 & 87.60 & 93.35 & 96.45 \\
\hline & $\left.F\left(\xi_{\mathrm{dB}}\right) ; F\left(\gamma_{\mathrm{dB}}\right)\right]+\mathrm{BCE}$ & 61.00 & 76.30 & 86.98 & 93.32 & 96.52 \\
\hline \multirow{9}{*}{ 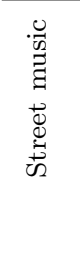 } & Noisy speech & 59.31 & 70.57 & 80.58 & 88.13 & 93.16 \\
\hline & $\mathrm{IRM}+\mathrm{MSE}$ & 69.74 & 80.81 & 88.28 & 92.78 & 95.42 \\
\hline & $\mathrm{IRM}+\mathrm{BCE}$ & 69.41 & 81.03 & 88.40 & 92.83 & 95.49 \\
\hline & $|S|^{\prime}+\mathrm{BCE}$ & 70.92 & 81.39 & 88.09 & 92.38 & 94.91 \\
\hline & $\left|S_{\mathrm{dB}}\right|+\mathrm{MSE}$ & 64.79 & 76.88 & 85.19 & 89.57 & 91.86 \\
\hline & $F\left(\xi_{\mathrm{dB}}\right)+\mathrm{MSE}$ & 66.20 & 79.28 & 87.50 & 92.68 & 95.47 \\
\hline & $F\left(\xi_{\mathrm{dB}}\right)+\mathrm{BCE}$ & 65.62 & 78.92 & 87.52 & 92.55 & 95.45 \\
\hline & $\left.F\left(\xi_{\mathrm{dB}}\right) ; F\left(\gamma_{\mathrm{dB}}\right)\right]+\mathrm{MSE}$ & 66.56 & 79.46 & 87.62 & 92.49 & 95.41 \\
\hline & $\left.F\left(\xi_{\mathrm{dB}}\right) ; F\left(\gamma_{\mathrm{dB}}\right)\right]+\mathrm{BCE}$ & 65.91 & 78.29 & 87.11 & 92.61 & 95.45 \\
\hline \multirow{9}{*}{$\frac{0}{I_{1}}$} & Noisy speech & 58.95 & 70.55 & 80.98 & 88.76 & 93.78 \\
\hline & IRM+MSE & 73.52 & 84.01 & 90.37 & 94.21 & 96.63 \\
\hline & IRM+BCE & 73.56 & 83.76 & 89.95 & 94.06 & 96.56 \\
\hline & $|S|^{\prime}+\mathrm{BCE}$ & 75.01 & 84.17 & 89.97 & 93.69 & 95.90 \\
\hline & $\left|S_{\mathrm{dB}}\right|+\mathrm{MSE}$ & 68.46 & 80.56 & 87.25 & 91.34 & 93.63 \\
\hline & $F\left(\xi_{\mathrm{dB}}\right)+\mathrm{MSE}$ & 70.41 & 82.47 & 89.65 & 93.91 & 96.43 \\
\hline & $F\left(\xi_{\mathrm{dB}}\right)+\mathrm{BCE}$ & 70.78 & 82.30 & 89.47 & 93.83 & 96.43 \\
\hline & $\left.F\left(\xi_{\mathrm{dB}}\right) ; F\left(\gamma_{\mathrm{dB}}\right)\right]+\mathrm{MSE}$ & 70.64 & 82.79 & 89.55 & 93.81 & 96.40 \\
\hline & $\left.F\left(\xi_{\mathrm{dB}}\right) ; F\left(\gamma_{\mathrm{dB}}\right)\right]+\mathrm{BCE}$ & 68.71 & 81.71 & 89.15 & 93.70 & 96.45 \\
\hline \multirow{9}{*}{ 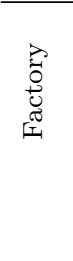 } & Noisy speech & 54.03 & 65.54 & 76.94 & 86.34 & 92.68 \\
\hline & IRM+MSE & 67.61 & 79.43 & 87.64 & 92.76 & 95.85 \\
\hline & IRM+BCE & 65.99 & 78.99 & 87.70 & 92.88 & 95.85 \\
\hline & $|S|^{\prime}+\mathrm{BCE}$ & 68.93 & 80.61 & 87.88 & 92.38 & 95.01 \\
\hline & $\left|S_{\mathrm{dB}}\right|+\mathrm{MSE}$ & 64.10 & 77.11 & 85.40 & 90.21 & 92.86 \\
\hline & $F\left(\xi_{\mathrm{dB}}\right)+\mathrm{MSE}$ & 64.65 & 77.86 & 87.05 & 92.46 & 95.48 \\
\hline & $F\left(\xi_{\mathrm{dB}}\right)+\mathrm{BCE}$ & 64.52 & 77.90 & 86.66 & 92.33 & 95.63 \\
\hline & {$\left[F\left(\xi_{\mathrm{dB}}\right) ; F\left(\gamma_{\mathrm{dB}}\right)\right]+\mathrm{MSE}$} & 63.29 & 77.04 & 86.42 & 91.99 & 95.42 \\
\hline & $\left.F\left(\xi_{\mathrm{dB}}\right) ; F\left(\gamma_{\mathrm{dB}}\right)\right]+\mathrm{BCE}$ & 63.20 & 76.40 & 86.08 & 91.91 & 95.36 \\
\hline
\end{tabular}


TABLE VIII. Mean WER for each condition of the test set from the Deep Xi dataset described in Subsection V C 2. The MMSE-LSA estimator is used by the MMSE training targets. The lowest WER for each condition is in boldface.

\begin{tabular}{|c|c|c|c|c|c|c|}
\hline \multirow{2}{*}{ Noise } & \multirow{2}{*}{ Target } & \multicolumn{5}{|c|}{ SNR } \\
\hline & & -5 & 0 & 5 & 10 & 15 \\
\hline $\begin{array}{l}0 \\
\frac{0}{0} \\
0 \\
0 \\
0 \\
0 \\
0 \\
0\end{array}$ & $\begin{array}{c}\text { Noisy speech } \\
\text { IRM+MSE } \\
\text { IRM+BCE } \\
|S|^{\prime}+\mathrm{BCE} \\
\left|S_{\mathrm{dB}}\right|+\mathrm{MSE} \\
F\left(\xi_{\mathrm{dB}}\right)+\mathrm{MSE} \\
F\left(\xi_{\mathrm{dB}}\right)+\mathrm{BCE} \\
{\left[F\left(\xi_{\mathrm{dB}}\right) ; F\left(\gamma_{\mathrm{dB}}\right)\right]+\mathrm{MSE}} \\
{\left[F\left(\xi_{\mathrm{dB}}\right) ; F\left(\gamma_{\mathrm{dB}}\right)\right]+\mathrm{BCE}}\end{array}$ & $\begin{array}{l}100.00 \\
91.60 \\
89.01 \\
92.98 \\
92.97 \\
90.89 \\
88.44 \\
\mathbf{8 7 . 5 6} \\
90.17\end{array}$ & $\begin{array}{l}89.17 \\
59.43 \\
60.29 \\
64.32 \\
64.24 \\
\mathbf{5 4 . 6 8} \\
65.56 \\
59.85 \\
58.99\end{array}$ & $\begin{array}{l}50.79 \\
32.12 \\
32.03 \\
31.22 \\
34.77 \\
30.08 \\
34.04 \\
\mathbf{2 8 . 3 2} \\
34.27\end{array}$ & $\begin{array}{l}21.07 \\
15.05 \\
13.57 \\
\mathbf{1 2 . 1 2} \\
20.03 \\
13.98 \\
14.52 \\
13.49 \\
14.45\end{array}$ & $\begin{array}{c}12.59 \\
\mathbf{9 . 2 1} \\
11.10 \\
11.36 \\
13.29 \\
9.93 \\
10.45 \\
10.13 \\
10.31\end{array}$ \\
\hline 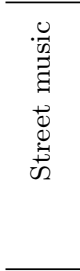 & $\begin{array}{c}\text { Noisy speech } \\
\text { IRM+MSE } \\
\text { IRM+BCE } \\
|S|^{\prime}+\mathrm{BCE} \\
\left|S_{\mathrm{dB}}\right|+\mathrm{MSE} \\
F\left(\xi_{\mathrm{dB}}\right)+\mathrm{MSE} \\
F\left(\xi_{\mathrm{dB}}\right)+\mathrm{BCE} \\
{\left[F\left(\xi_{\mathrm{dB}}\right) ; F\left(\gamma_{\mathrm{dB}}\right)\right]+\mathrm{MSE}} \\
{\left[F\left(\xi_{\mathrm{dB}}\right) ; F\left(\gamma_{\mathrm{dB}}\right)\right]+\mathrm{BCE}} \\
\end{array}$ & $\begin{array}{l}98.05 \\
83.57 \\
83.15 \\
81.86 \\
87.23 \\
\mathbf{8 0 . 2 8} \\
80.32 \\
83.75 \\
82.78\end{array}$ & $\begin{array}{l}88.60 \\
52.56 \\
55.16 \\
56.65 \\
61.21 \\
\mathbf{5 1 . 5 0} \\
61.17 \\
59.38 \\
53.33\end{array}$ & $\begin{array}{l}56.59 \\
\mathbf{2 4 . 8 8} \\
26.20 \\
25.67 \\
40.97 \\
29.06 \\
28.53 \\
29.21 \\
29.01\end{array}$ & $\begin{array}{l}26.24 \\
14.54 \\
16.09 \\
17.49 \\
23.50 \\
15.18 \\
14.67 \\
14.64 \\
\mathbf{1 4 . 0 0}\end{array}$ & $\begin{array}{l}16.16 \\
14.34 \\
13.75 \\
14.45 \\
14.24 \\
\mathbf{9 . 6 9} \\
13.79 \\
12.46 \\
13.79\end{array}$ \\
\hline$\stackrel{0}{\stackrel{1}{I}}$ & $\begin{array}{c}\text { Noisy speech } \\
\text { IRM+MSE } \\
\text { IRM+BCE } \\
|S|^{\prime}+\mathrm{BCE} \\
\left|S_{\mathrm{dB}}\right|+\mathrm{MSE} \\
F\left(\xi_{\mathrm{dB}}\right)+\mathrm{MSE} \\
F\left(\xi_{\mathrm{dB}}\right)+\mathrm{BCE} \\
{\left[F\left(\xi_{\mathrm{dB}}\right) ; F\left(\gamma_{\mathrm{dB}}\right)\right]+\mathrm{MSE}} \\
{\left[F\left(\xi_{\mathrm{dB}}\right) ; F\left(\gamma_{\mathrm{dB}}\right)\right]+\mathrm{BCE}}\end{array}$ & $\begin{array}{l}98.83 \\
89.38 \\
91.61 \\
\mathbf{8 4 . 4 9} \\
89.33 \\
95.83 \\
90.00 \\
90.66 \\
88.68\end{array}$ & $\begin{array}{l}99.60 \\
56.66 \\
50.67 \\
60.11 \\
60.10 \\
58.92 \\
61.19 \\
\mathbf{5 0 . 0 5} \\
54.66\end{array}$ & $\begin{array}{l}63.17 \\
\mathbf{2 3 . 9 4} \\
28.00 \\
30.34 \\
41.54 \\
27.62 \\
32.16 \\
24.82 \\
35.05\end{array}$ & $\begin{array}{l}30.14 \\
14.48 \\
15.88 \\
19.20 \\
21.88 \\
14.13 \\
\mathbf{1 3 . 0 8} \\
13.38 \\
14.20\end{array}$ & $\begin{array}{l}18.45 \\
14.19 \\
10.40 \\
16.79 \\
15.98 \\
13.83 \\
9.90 \\
\mathbf{9 . 3 9} \\
14.05\end{array}$ \\
\hline 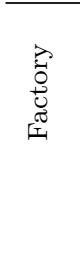 & $\begin{array}{c}\text { Noisy speech } \\
\text { IRM+MSE } \\
\text { IRM+BCE } \\
|S|^{\prime}+\mathrm{BCE} \\
\left|S_{\mathrm{dB}}\right|+\mathrm{MSE} \\
F\left(\xi_{\mathrm{dB}}\right)+\mathrm{MSE} \\
F\left(\xi_{\mathrm{dB}}\right)+\mathrm{BCE} \\
{\left[F\left(\xi_{\mathrm{dB}}\right) ; F\left(\gamma_{\mathrm{dB}}\right)\right]+\mathrm{MSE}} \\
{\left[F\left(\xi_{\mathrm{dB}}\right) ; F\left(\gamma_{\mathrm{dB}}\right)\right]+\mathrm{BCE}}\end{array}$ & $\begin{array}{l}97.66 \\
\mathbf{9 0 . 7 9} \\
90.82 \\
93.78 \\
93.12 \\
92.35 \\
94.96 \\
91.76 \\
91.57\end{array}$ & $\begin{array}{l}97.14 \\
65.78 \\
68.79 \\
\mathbf{6 3 . 1 4} \\
81.19 \\
77.96 \\
73.41 \\
67.23 \\
77.00\end{array}$ & $\begin{array}{l}66.70 \\
47.02 \\
49.43 \\
53.06 \\
60.41 \\
62.36 \\
47.19 \\
\mathbf{4 5 . 5 7} \\
58.66\end{array}$ & $\begin{array}{l}31.86 \\
25.69 \\
28.17 \\
\mathbf{2 3 . 7 3} \\
37.97 \\
28.07 \\
25.63 \\
30.16 \\
31.09\end{array}$ & $\begin{array}{c}14.83 \\
6.38 \\
\mathbf{5 . 3 0} \\
9.17 \\
22.81 \\
8.25 \\
14.52 \\
15.70 \\
11.92\end{array}$ \\
\hline
\end{tabular}

${ }^{1}$ Note that parameters required for each compression function are omitted for convenience. For example $z\left(\left|S_{\mathrm{dB}}[l, k]\right| ; \mu_{k}, \sigma_{k}\right)$ is simplified to $z\left(\left|S_{\mathrm{dB}}[l, k]\right|\right)$.

${ }^{2}$ Deep Xi is available at: https://github.com/anicolson/DeepXi. ${ }^{3}$ For a comparison of the ResNet TCN used here to that used in (Zhang et al., 2020), please see https://github.com/anicolson/ DeepXi.

${ }^{4}$ Freesound packs that are used include 147, 199, 247, 379, 622, 643, $1133,1563,1840,2432,4366,4439,4780,8420,14826,15046$, 15 097, 15 598, 16 204, $17266,17403,17430,17468,17579,19093$, $20237,20241,21558,22953$, and 24590.

${ }^{5}$ Project DeepSpeech is available at: https://github.com/ mozilla/DeepSpeech (model 0.7.4 is used).

Abadi, M., Agarwal, A., Barham, P., Brevdo, E., Chen, Z., Citro, C., Corrado, G. S., Davis, A., Dean, J., Devin, M., Ghemawat, S., Goodfellow, I., Harp, A., Irving, G., Isard, M., Jia, Y., Jozefowicz, R., Kaiser, L., Kudlur, M., Levenberg, J., Mané, D., Monga, R., Moore, S., Murray, D., Olah, C., Schuster, M., Shlens, J., Steiner, B., Sutskever, I., Talwar, K., Tucker, P., Vanhoucke, V., Vasudevan, V., Viégas, F., Vinyals, O., Warden, P., Wattenberg, M., Wicke, M., Yu, Y., and Zheng, X. (2015). "TensorFlow: Large-scale machine learning on heterogeneous systems" https://www.tensorflow.org/, software available from tensorflow.org.
Allen, J. (1977). "Short term spectral analysis, synthesis, and modification by discrete Fourier transform," IEEE Transactions on Acoustics, Speech, and Signal Processing 25(3), 235-238.

Allen, J. B., and Rabiner, L. R. (1977). "A unified approach to short-time Fourier analysis and synthesis," Proceedings of the IEEE 65(11), 1558-1564.

Ba, J. L., Kiros, J. R., and Hinton, G. E. (2016). "Layer normalization," arXiv:1607.06450 [stat.ML].

Cappe, O. (1994). "Elimination of the musical noise phenomenon with the ephraim and malah noise suppressor," IEEE Transactions on Speech and Audio Processing 2(2), 345-349.

Cohen, I. (2003). "Noise spectrum estimation in adverse environments: Improved minima controlled recursive averaging," IEEE Transactions on Speech and Audio Processing 11(5), 466-475.

Crochiere, R. (1980). "A weighted overlap-add method of shorttime Fourier analysis/synthesis," IEEE Transactions on Acoustics, Speech, and Signal Processing 28(1), 99-102.

Dean, D. B., Sridharan, S., Vogt, R. J., and Mason, M. W. (2010). "The QUT-NOISE-TIMIT corpus for the evaluation of voice activity detection algorithms," in In INTERSPEECH-2010, pp. $3110-3113$.

Ephraim, Y., and Malah, D. (1984). "Speech enhancement using a minimum-mean square error short-time spectral amplitude estimator," IEEE Transactions on Acoustics, Speech, and Signal Processing 32(6), 1109-1121.

Ephraim, Y., and Malah, D. (1985). "Speech enhancement using a minimum mean-square error log-spectral amplitude estimator," IEEE Transactions on Acoustics, Speech, and Signal Processing 33(2), 443-445.

Erdogan, H., Hershey, J. R., Watanabe, S., and Le Roux, J. (2015). "Phase-sensitive and recognition-boosted speech separation using deep recurrent neural networks," in 2015 IEEE International Conference on Acoustics, Speech and Signal Processing (ICASSP), pp. 708-712.

Hannun, A., Case, C., Casper, J., Catanzaro, B., Diamos, G., Elsen, E., Prenger, R., Satheesh, S., Sengupta, S., Coates, A. et al. (2014). "Deep speech: Scaling up end-to-end speech recognition," arXiv:1412.5567 [cs.CL] .

He, K., Zhang, X., Ren, S., and Sun, J. (2016). "Deep residual learning for image recognition," in The IEEE Conference on Computer Vision and Pattern Recognition (CVPR).

$\mathrm{Hu}$, G., and Wang, D. (2010). "A tandem algorithm for pitch estimation and voiced speech segregation," IEEE Transactions on Audio, Speech, and Language Processing 18(8), 2067-2079.

Hu, Y., and Loizou, P. C. (2008). "Evaluation of objective quality measures for speech enhancement," IEEE Transactions on Audio, Speech, and Language Processing 16(1), 229-238.

Kim, J., El-Khamy, M., and Lee, J. (2017). "Residual LSTM: Design of a deep recurrent architecture for distant speech recognition," in Proc. Interspeech 2017, pp. 1591-1595, http:// dx.doi.org/10.21437/Interspeech.2017-477, doi: 10.21437/ Interspeech.2017-477.

Kingma, D. P., and Ba, J. (2014). "Adam: A method for stochastic optimization," arXiv:1412.6980 [cs.LG]

Kriman, S., Beliaev, S., Ginsburg, B., Huang, J., Kuchaiev, O., Lavrukhin, V., Leary, R., Li, J., and Zhang, Y. (2020). "Quartznet: Deep automatic speech recognition with 1D timechannel separable convolutions," in ICASSP 2020 - 2020 IEEE International Conference on Acoustics, Speech and Signal Processing (ICASSP), pp. 6124-6128.

Le Prell, C. G., and Clavier, O. H. (2017). "Effects of noise on speech recognition: Challenges for communication by service members," Hearing Research 349, 76 - 89, http://www.sciencedirect.com/science/article/pii/ S0378595516303513, doi: https://doi.org/10.1016/j.heares. 2016.10.004 noise in the Military.

Lim, J. S., and Oppenheim, A. V. (1979). "Enhancement and bandwidth compression of noisy speech," Proceedings of the IEEE 67(12), 1586-1604.

Loizou, P. C. (2005). "Speech enhancement based on perceptually motivated Bayesian estimators of the magnitude spectrum," IEEE Transactions on Speech and Audio Processing 13(5), 857869.

Loizou, P. C. (2013). Speech Enhancement: Theory and Practice, 2nd ed. (CRC Press, Inc., USA). 
Martin, R. (2005). "Speech enhancement based on minimum mean-square error estimation and supergaussian priors," IEEE Transactions on Speech and Audio Processing 13(5), 845-856.

Mermelstein, P. (1979). "Evaluation of a segmental SNR measure as an indicator of the quality of ADPCM coded speech," The Journal of the Acoustical Society of America 66(6), 1664-1667, https://doi.org/10.1121/1.383638, doi: 10.1121/1.383638.

Morioka, C., Kurashima, A., and Takahashi, A. (2005). "Proposal on objective speech quality assessment for wideband IP telephony," in Proceedings. (ICASSP '05). IEEE International Conference on Acoustics, Speech, and Signal Processing, 2005. Vol. 1 , pp. $49-52$

Moritz, N., Hori, T., and Le, J. (2020). "Streaming automatic speech recognition with the transformer model," in ICASSP 2020 - 2020 IEEE International Conference on Acoustics, Speech and Signal Processing (ICASSP), pp. 6074-6078.

Narayanan, A., and Wang, D. (2013). "Ideal ratio mask estimation using deep neural networks for robust speech recognition," in 2013 IEEE International Conference on Acoustics, Speech and Signal Processing, pp. 7092-7096.

Nicolson, A. (2020). "Deep Xi training set," IEEE Dataport http: //dx.doi.org/10.21227/3adt-pb04, doi: 10.21227/3adt-pb04.

Nicolson, A., and Paliwal, K. K. (2019a). "Deep learning for minimum mean-square error approaches to speech enhancement," Speech Communication 111, 44 - 55, http://www. sciencedirect.com/science/article/pii/S0167639318304308, doi: https://doi.org/10.1016/j.specom.2019.06.002.

Nicolson, A., and Paliwal, K. K. (2019b). "Deep Xi as a frontend for robust automatic speech recognition," arXiv:1906.07319 [eess.AS] .

Nicolson, A., and Paliwal, K. K. (2020). "Sum-product networks for robust automatic speaker identification," in Proc. Interspeech 2020.

Nikzad, M., Nicolson, A., Gao, Y., Zhou, J., Paliwal, K. K., and Shang, F. (2020). "Deep residual-dense lattice network for speech enhancement," in The Thirty-Fourth AAAI Conference on Artificial Intelligence (AAAI-20), pp. 8552-8559.

Panayotov, V., Chen, G., Povey, D., and Khudanpur, S. (2015). "Librispeech: An asr corpus based on public domain audio books," in 2015 IEEE International Conference on Acoustics, Speech and Signal Processing (ICASSP), pp. 5206-5210.

Pascual, S., Bonafonte, A., and Serrà, J. (2017). "SEGAN: Speech enhancement generative adversarial network," in Proc. Interspeech 2017, pp. 3642-3646, http://dx.doi.org/10. 21437/Interspeech.2017-1428, doi: 10.21437/Interspeech. 2017-1428.

Rix, A. W., Beerends, J. G., Hollier, M. P., and Hekstra, A. P. (2001). "Perceptual evaluation of speech quality (PESQ)-a new method for speech quality assessment of telephone networks and codecs," in 2001 IEEE International Conference on Acoustics, Speech, and Signal Processing. Proceedings (Cat. No.01CH37221), Vol. 2, pp. 749-752 vol.2.

Saki, F., Sehgal, A., Panahi, I., and Kehtarnavaz, N. (2016). "Smartphone-based real-time classification of noise signals using subband features and random forest classifier," in 2016 IEEE International Conference on Acoustics, Speech and Signal Processing (ICASSP), pp. 2204-2208.

Salamon, J., Jacoby, C., and Bello, J. P. (2014). "A dataset and taxonomy for urban sound research," in Proceedings of the 22nd ACM International Conference on Multimedia, MM '14, Association for Computing Machinery, New York, NY, USA, p. 1041-1044, https://doi.org/10.1145/2647868.2655045, doi: 10.1145/2647868.2655045

Schluter, R., Bezrukov, I., Wagner, H., and Ney, H. (2007). "Gammatone features and feature combination for large vocabulary speech recognition," in 2007 IEEE International Conference on Acoustics, Speech and Signal Processing - ICASSP '07, Vol. 4, pp. IV-649-IV-652.

Snyder, D., Chen, G., and Povey, D. (2015). "MUSAN: A music, speech, and noise corpus," arXiv:1510.08484 [cs.SD] .

Srinivasan, S., Roman, N., and Wang, D. (2006). "Binary and ratio time-frequency masks for robust speech recognition," Speech Communication 48(11), 1486 - 1501, http://www. sciencedirect.com/science/article/pii/S0167639306001129, doi: https://doi.org/10.1016/j.specom.2006.09.003 robust- ness Issues for Conversational Interaction.

Steeneken, H. J., and Geurtsen, F. W. (1988). "Description of the RSG-10 noise database," Report IZF 1988-3, TNO Institute for Perception, Soesterberg, The Netherlands

Taal, C. H., Hendriks, R. C., Heusdens, R., and Jensen, J. (2010). "A short-time objective intelligibility measure for time-frequency weighted noisy speech," in 2010 IEEE International Conference on Acoustics, Speech and Signal Processing, pp. 4214-4217.

Taal, C. H., Hendriks, R. C., Heusdens, R., and Jensen, J. (2011). "An algorithm for intelligibility prediction of time-frequency weighted noisy speech," IEEE Transactions on Audio, Speech, and Language Processing 19(7), 2125-2136.

Tawara, N., Kobayashi, T., and Ogawa, T. (2019). "Multi-Channel Speech Enhancement Using Time-Domain Convolutional Denoising Autoencoder," in Proc. Interspeech 2019, pp. 86-90, http:// dx.doi.org/10.21437/Interspeech.2019-3197, doi: 10.21437/ Interspeech.2019-3197.

Thiemann, J., Ito, N., and Vincent, E. (2013). "The diverse environments multi-channel acoustic noise database (DEMAND): A database of multichannel environmental noise recordings," Proceedings of Meetings on Acoustics 19(1), 035081, https://asa. scitation.org/doi/abs/10.1121/1.4799597, doi: 10.1121/1. 4799597.

Thomas-Stonell, N., Kotler, A.-L., Leeper, H., and Doyle, P. (1998). "Computerized speech recognition: Influence of intelligibility and perceptual consistency on recognition accuracy," Augmentative and Alternative Communication 14(1), 51-56, https://doi.org/10.1080/07434619812331278196, doi: $10.1080 / 07434619812331278196$

Valentini-Botinhao, C., Wang, X., Takaki, S., and Yamagishi, J. (2016). "Investigating RNN-based speech enhancement methods for noise-robust text-to-speech," in 9th ISCA Speech Synthesis Workshop, pp. 146-152, http://dx.doi.org/10.21437/SSW. 2016-24, doi: 10.21437/SSW.2016-24.

Vary, P., and Martin, R. (2006). Digital Speech Transmission: Enhancement, Coding And Error Concealment (John Wiley \& Sons, Inc., Hoboken, NJ, USA).

Veaux, C., Yamagishi, J., and King, S. (2013). "The voice bank corpus: Design, collection and data analysis of a large regional accent speech database," in 2013 International Conference Oriental COCOSDA held jointly with 2013 Conference on Asian Spoken Language Research and Evaluation ( $O$ COCOSDA/CASLRE), pp. 1-4.

Veaux, C., Yamagishi, J., and MacDonald, K. (2017). "CSTR VCTK corpus: English multi-speaker corpus for CSTR voice cloning toolkit," Technical Report.

Wang, D. (2005). "On ideal binary mask as the computational goal of auditory scene analysis," in Speech Separation by Humans and Machines, edited by P. Divenyi (Springer US, Boston, MA), pp. 181-197, https://doi.org/10.1007/0-387-22794-6_12, doi: 10.1007/0-387-22794-6_12.

Wang, D., and Brown, G. J. (2006). Computational Auditory Scene Analysis: Principles, Algorithms, and Applications (Wiley-IEEE Press).

Wang, Y., Mohamed, A., Le, D., Liu, C., Xiao, A., Mahadeokar, J., Huang, H., Tjandra, A., Zhang, X., Zhang, F., Fuegen, C., Zweig, G., and Seltzer, M. L. (2020). "Transformer-based acoustic modeling for hybrid speech recognition," in ICASSP 2020 2020 IEEE International Conference on Acoustics, Speech and Signal Processing (ICASSP), pp. 6874-6878.

Wang, Y., Narayanan, A., and Wang, D. (2014). "On training targets for supervised speech separation," IEEE/ACM Transactions on Audio, Speech, and Language Processing 22(12), 1849-1858.

Wang, Y., and Wang, D. (2013). "Towards scaling up classification-based speech separation," IEEE Transactions on Audio, Speech, and Language Processing 21(7), 1381-1390.

Williamson, D. S., Wang, Y., and Wang, D. (2016). "Complex ratio masking for monaural speech separation," IEEE/ACM Transactions on Audio, Speech, and Language Processing 24(3), 483492

Xu, J., Sun, X., Zhang, Z., Zhao, G., and Lin, J. (2019). "Understanding and improving layer normalization," in Advances in Neural Information Processing Systems 32, edited by $\mathrm{H}$. Wallach, H. Larochelle, A. Beygelzimer, F. d'Alché Buc, E. Fox, and R. Garnett (Curran Asso- 
ciates, Inc.), pp. 4381-4391, http://papers.nips.cc/paper/ 8689-understanding-and-improving-layer-normalization. pdf.

Xu, Y., Du, J., Dai, L., and Lee, C. (2015). "A regression approach to speech enhancement based on deep neural networks," IEEE/ACM Transactions on Audio, Speech, and Language Processing 23(1), 7-19.

Zhang, Q., Nicolson, A., Wang, M., Paliwal, K. K., and Wang, C. (2020). "DeepMMSE: A deep learning approach to MMSE-based noise power spectral density estimation," IEEE/ACM Transactions on Audio, Speech, and Language Processing 28, 1404-1415.
Zhang, Z., Geiger, J., Pohjalainen, J., Mousa, A. E.-D., Jin, W. and Schuller, B. (2018). "Deep learning for environmentally robust speech recognition: An overview of recent developments," ACM Trans. Intell. Syst. Technol. 9(5), https://doi.org/10. 1145/3178115, doi: 10.1145/3178115.

Zhao, Y., Wang, Z., and Wang, D. (2017). "A two-stage algorithm for noisy and reverberant speech enhancement," in 2017 IEEE International Conference on Acoustics, Speech and Signal Processing (ICASSP), pp. 5580-5584. 